\title{
Instability of cooperation in finite populations
}

\author{
Chai Molina ${ }^{1 a, b, c}$ and David J. D. Earn ${ }^{c}$ \\ ${ }^{a}$ Department of Ecology and Evolutionary Biology, Princeton University, Princeton 08544 NJ, USA \\ ${ }^{b}$ International Institute for Applied Systems Analysis, Laxenburg, A-2361, Austria \\ ${ }^{\mathrm{c}}$ Department of Mathematics \& Statistics, McMaster University, Hamilton ON L8S 4K1, Canada
}

\begin{abstract}
Evolutionary game theory has been developed primarily under the implicit assumption of an infinite population. We rigorously analyze a standard model for the evolution of cooperation (the multi-player snowdrift game) and show that in many situations in which there is a cooperative evolutionarily stable strategy (ESS) if the population is infinite, there is no cooperative ESS if the population is finite (no matter how large). In these cases, contributing nothing is a globally convergently stable finite-population ESS, implying that apparent evolution of cooperation in such games is an artifact of the infinite population approximation. The key issue is that if the size of groups that play the game exceeds a critical proportion of the population then the infinite-population approximation predicts the wrong evolutionary outcome (in addition, the critical proportion itself depends on the population size). Our results are robust to the underlying selection process.
\end{abstract}

cooperation | public goods games | evolutionary game theory

ORCID IDs of authors: CM 0000-0001-9722-4446; DE 0000-0002-7562-1341

\footnotetext{
${ }^{1}$ Corresponding author; e-mail: chai.molina@gmail.com
} 
 \\ 1 Introduction}

Many evolutionary games assume - for mathematical convenience - that populations are infinitely large (e.g., (1-7)). This assumption is sometimes justified on the grounds that "[p]opulations which stay numerically small quickly go extinct by chance fluctuations" (8, §2.1). Of course, all real populations are finite, and important differences in evolutionary dynamics between finite and infinite populations have been demonstrated (9-15). In spite of the technical challenges of working with finite populations, some exact analytical results have been obtained for two-player games with discrete strategy sets (9, 12, 14-16). However, most existing finite-population results rely on approximation methods and simulations (11, 15, 17-21). Notably, almost all finite-population results involve discrete strategy sets, such as when individuals must choose between making a fixed positive contribution to a public good, or nothing at all (e.g., $(9,12,14-16))$. Yet, evolutionary games involving continuous strategy sets (e.g., allocating time or effort to a communal task) are both widely applicable and extensively studied using infinite-population models (22). Moreover, to our knowledge, all existing results for finite populations depend on a choice of selection process (e.g., Moran or Wright-Fisher $(23,24)$ ).

Here, we present mathematically rigorous results that identify critical differences in the predictions of evolutionary games in finite and infinite populations. We focus on a standard model for exploring the evolution of cooperation - the continuous multi-player snowdrift game (3) - which has previously been studied in infinite populations using exact analysis and simulations $(3,7,25-27)$ and in finite populations using approximations and simulations $(11,21,28,29)$.

We show that evolutionary outcomes in finite and infinite populations can be dramatically different. In particular, for a class of snowdrift games for which a cooperative ESS exists in infinite populations (30), we find conditions under which there is no cooperative ESS when played in finite populations. This qualitative difference in predictions for finite and infinite populations can occur no matter how large the finite population is, and is universal in the sense that it is independent of the selection process (31). To our knowledge, there are no other examples in the literature of qualitatively different dynamics in finite and infinite populations that persist for arbitrarily large populations and are independent of the selection process; other studies that demonstrate such differences (e.g., (32)) are restricted to particular selection processes.

The results we present are supported by formal mathematical theorems, which we state in Results and prove in Methods and Supporting Information.

\section{Terminology}

The snowdrift game is an abstraction of the situation in which a group of individuals encounters a snowdrift that blocks their path. We suppose that $n$ players are drawn from a population of self-interested individuals ( $n$ is the group size), and that each player chooses how much to contribute to a public good-e.g., snow cleared off the path - from which all group members benefit. A focal individual contributing $x$ incurs a cost $C(x)$ that depends only on its own contribution, whereas its benefit $B(\tau)$ depends on the total good $\tau$ contributed by the group as a whole. The focal individual's payoff - which is interpreted as a 
change in fitness - is then

$$
W(x, \tau)=B(\tau)-C(x) .
$$

If $x$ is a continuous variable, as we assume here, the game is said to be continuous. Positive contributions represent cooperative strategies, and individuals who contribute nothing are said to defect. If the population is finite and contains $N$ individuals, then for convenience we refer to the ratio $G=N / n$ as the number of groups; however, we do not assume that the population is simultaneously subdivided into groups of $n$ individuals (and in particular, $G$ need not be an integer).

To avoid mathematical complexities that are not relevant to the biological issues that concern us, we impose a few natural conditions on the cost and benefit functions and refer to the natural snowdrift game (NSG; see Methods §5.1). The NSG was introduced in (30), where it was shown that - when played in infinite populations - the game always has a cooperative ESS. Cost, benefit and fitness functions for an NSG example are shown in figure 1.

Traditionally, an evolutionarily stable strategy (ESS) is one such that, when adopted by the entire population, a single mutant individual playing a different strategy cannot invade the population (33). Because the phenotypic changes caused by mutations are often small, local ESSs are of particular interest: a population of individuals playing a local ESS is resistant to the invasion of a single individual playing a slightly different strategy. A strategy is convergently stable if a population playing a different strategy evolves toward it (34); convergence can be either global or local.

In infinite populations, the theory of adaptive dynamics $(2,8,35)$ identifies a singular strategy as one at which the selection gradient, $\left.\partial_{x} W(x, x+(n-1) X)\right|_{x=X}$, vanishes (36, Table 1); for an NSG, this reduces to

$$
B^{\prime}(n X)-1=0 .
$$

A singular strategy for which the mutant fitness is concave near the singular strategy is a local ESS.* Local convergent stability of singular strategies is also defined via a condition on the local fitness difference [see Table 1 of (2)].

The definition of singular strategies can be extended to finite populations: The defining feature of a singular strategy is that when it is played by a resident population, directional selection vanishes; for an NSG, this condition reduces to

$$
\frac{N-n}{N-1} B^{\prime}(n X)-1=0
$$

[see definition 4.3.5 of (37) and equation (28)]. The finite-population extension of the concept of evolutionary stability is more involved, because it must account for the fact that selection can favour fixation of a mutant strategy, even if selection opposes its invasion (9). Thus, the standard definition of an evolutionary stable strategy in a finite population (ESS (9)) requires that selection oppose both invasion by, and fixation of, mutant strategies. In addition, the presence of one or more mutants in a finite population has a non-negligible effect on the fitness of residents (whereas finitely many mutants cannot affect the mean fitness of residents in an infinite population).

\footnotetext{
${ }^{*}$ We assume throughout this paper that the strategy space is one-dimensional.
} 

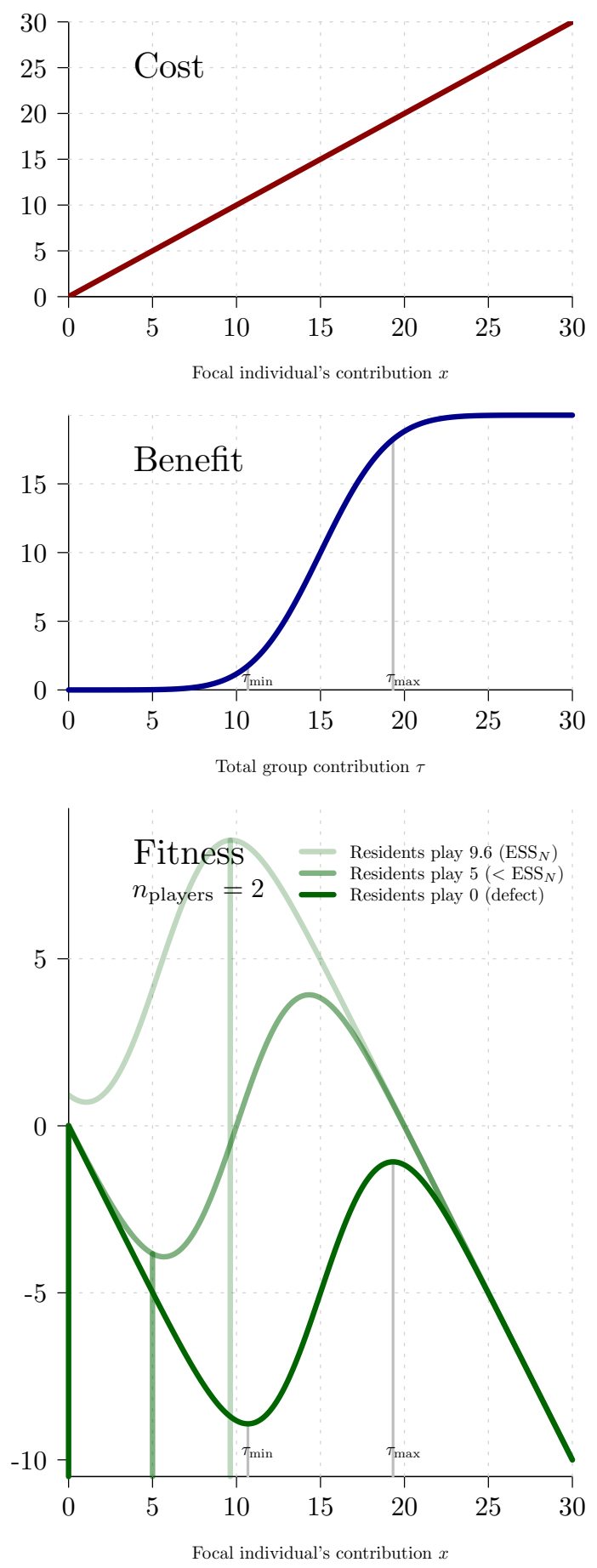

Figure 1: Example cost, benefit and fitness functions for a natural snowdrift game (NSG, defined in Methods §5.1). Top panel: The cost function is simply $C(x)=x$. Middle panel: The benefit function $B(\tau)$ is given in Methods equation (23); parameter values are $L=10, k=1, m=$ 1.5, $\tau_{\text {turn }}=15$. Bottom panel: Fitness is shown for three situations involving groups of $n=$ 2 individuals. (i) Residents cooperate and contribute the $\mathrm{ESS}_{\mathrm{N}}$ (light green, $X_{\text {res }}=9.63$ ) (ii) Residents cooperate but contribute less than the $\mathrm{ESS}_{\mathrm{N}}$ (medium green, $X_{\text {res }}=5$ ). (iii) Residents defect, i.e., contribute nothing (dark green, $X_{\text {res }}=0$ ). Resident strategies are indicated by vertical lines in the same colour as the associated fitness function. In the case of defecting residents, a focal individual's fitness function does not depend on the group size $(n)$ and has a local maximum at the maximizing total good $\left(\tau_{\max }=19.3\right.$, thin grey vertical line). 
Fixation probabilities depend on the selection process (31), i.e., the stochastic process by which differences in fitnesses of individuals playing different strategies generate changes in the frequencies of strategies in the population over time. As a result, the strategies that are evolutionarily stable in finite populations depend on the selection process. Variants of the Moran and Wright-Fisher processes $(23,38,39)$ are commonly assumed, but are idealizations that do not exactly describe realistic populations (e.g., (40)). We are spared this complication in this paper because, for the games we consider, every ESS is a universal ESS, that is, all ESSs are evolutionarily stable irrespective of the selection process. Consequently, we need not specify the population-genetic processes underlying selection, and we obtain general results about evolutionary stability. We use the term universal more generally to indicate that a property or statement holds for any selection process.

\section{Results}

ESSs in infinite populations. As we have previously shown (30), if an NSG (Methods §5.1) is played in an infinite population then there are always two (and only two) ESSs:

defect: contribute nothing $(x=0)$, or

cooperate: make a positive contribution that is inversely proportional to the group size $n\left(x=X_{\infty}^{*}>0\right)$.

Both ESSs are global, and both are locally convergently stable [theorem 4.1 of (30)]. At the cooperative ESS, everyone contributes an equal share of the amount that maximizes individual fitness given that everyone contributes equally. In terms of this maximizing total good $\tau_{\max }$ (see Methods $§ 5.1$ and figure 1 ), the cooperative ESS is

$$
X_{\infty}^{*}=\frac{\tau_{\max }}{n} .
$$

ESSs in finite populations. In a finite population, NSGs do not necessarily have a cooperative $\mathrm{ESS}_{\mathrm{N}}$, and when they do it is not necessarily possible to find an explicit formula for evolutionarily stable cooperation levels in terms of the parameters of an NSG (nevertheless, cooperative $\mathrm{ESS}_{\mathrm{N}} \mathrm{S}$ are always easy to find numerically within the interval (6) identified in the following theorem).

Theorem 1 (Existence and universality of stable cooperation levels in the natural snowdrift game). Consider a finite population (of $N$ individuals) that is subject to selection resulting from groups of $n$ individuals playing an NSG [defined in Methods §5.1]. A strategy $X$ is singular if and only if

$$
B^{\prime}(n X)=1+\frac{n-1}{N-n},
$$

and any such strategy $X$ lies in the open interval

$$
\left(\frac{\tau_{\min }}{n}, \frac{\tau_{\max }}{n}\right) .
$$


such that ${ }^{\dagger}$

$$
\begin{gathered}
m>m_{\mathrm{c}} \Longrightarrow\left\{\begin{array}{l}
\text { Generically, at least one cooperative local ES } S_{N}(X= \\
\left.X_{N}^{*}>0\right) \text { exists that is universal and universally locally } \\
\text { convergently stable. In addition, defection is (univer- } \\
\text { sally) a locally convergently stable ES } S_{N} .
\end{array}\right. \\
m=m_{\mathrm{c}} \Longrightarrow\left\{\begin{array}{l}
\text { Generically, no cooperative ES } S_{N} \text { exists. Defection is } \\
\text { (universally) globally evolutionarily stable and locally } \\
\text { convergently stable. }
\end{array}\right. \\
m<m_{\mathrm{c}} \Longrightarrow\left\{\begin{array}{l}
\text { No cooperative ES } S_{N} \text { exists. Defection is (universally) } \\
\text { globally evolutionarily and convergently stable. }
\end{array}\right.
\end{gathered}
$$

Then $m>0$ and there is a critical maximal marginal fitness threshold,

$$
m_{\mathrm{c}}=\frac{N-1}{N-n}-1
$$

\section{then $X$ is a universal $E S S_{N}$ that is (universally) locally convergently stable.}

$\mathbf{E S S}_{\mathbf{N}} \mathbf{S}$ in large populations: If $B^{\prime \prime}\left(\tau_{\max }\right) \neq 0$ and the group size $n$ is either fixed, or satisfies $\frac{n(N)}{N} \stackrel{N \rightarrow \infty}{\longrightarrow} 0$, then for any sufficiently large population size $N$, there is a universal $E S S_{N} X=X_{N}^{*}$ satisfying inequality (8). Moreover, $X_{N}^{*} \rightarrow X_{\infty}^{*}$ as $N \rightarrow \infty$.

While the evolutionarily stable cooperation levels in finite and infinite populations are never xactly the same, theorem 1 shows that the difference is negligible in sufficiently large populations if as the population size $N \rightarrow \infty$, groups become a vanishingly small proportion of the population ( $c f$. figure 2). However, if group size is not sufficiently small relative to the otal population size then evolutionary predictions from finite population models differ qualtatively from the predictions for infinite ones: it may actually be impossible for cooperation Theorem $2\left(\mathrm{ESS}_{\mathrm{N}} \mathrm{S}\right.$ of the natural snowdrift game). Consider a finite population (of $N$ individuals) that is subject to selection resulting from groups of $n$ individuals playing an $N S G$ [defined in Methods $\$ 5.1$ with fitness $W$ defined by equation (20)]. Let $m$ denote the maximal marginal fitness, i.e.,

$$
m \equiv \max _{\tau \geq 0}\left(\frac{\partial W}{\partial x}\right)=\max _{\tau \geq 0}\left(B^{\prime}(\tau)-1\right) .
$$

In (11a), "generically" means excluding the unlikely possibility of singular strategies also being inflection points of $B(n x)$; in (11b), it excludes the possibility of the marginal benefit $B^{\prime}(\tau)$ being constant in a neighbourhood of $\arg \max B^{\prime}(\tau)$. 
This theorem predicts qualitatively different evolutionary outcomes, depending on the maximal marginal fitness $(m)$ : Equation $(10)$ gives the critical maximal marginal fitness above which a cooperative $\mathrm{ESS}_{\mathrm{N}}$ exists, and below which defection is the only $\mathrm{ESS}_{\mathrm{N}}$. Theorem 2 thus connects the maximal marginal fitness - a property of the fitness function that relates investments in the communal task to fitness benefits - with properties of the population of interacting agents: the population size $(N)$, the number of players in a group $(n)$, and the number of groups $(G=N / n)$.

Equation (10) expresses the critical maximal marginal fitness in terms of a given population size and given group size. To clarify the roles of group size and number of groups in the evolution of cooperation, it is useful to think instead of the maximal marginal fitness $(m)$ as given (i.e., as a fixed property of the strategic interaction). Then, in the inequality $m>m_{\mathrm{c}}$ [see (11a)], we can replace $m_{\mathrm{c}}$ by the expression on the right hand size of Equation (10), and solve for a critical number of groups $\left(G_{\mathrm{c}}\right)$ or critical group size $\left(n_{\mathrm{c}}\right)$.

ESS conditions in relation to the number of groups $(G)$. Condition (11a) can be expressed equivalently as

$$
G>G_{\mathrm{c}} \equiv \frac{m+1}{m+(1 / N)}=1+\frac{1}{m}\left(1-\frac{1}{n}\right)
$$

i.e., the number of groups $G$ must be greater than $G_{\mathrm{c}}$, the minimum number of groups that support cooperation in a population of size $N$ (or in groups of $n$ players). For any given number of players in a group $(n)$, if we multiply inequality (12) by $n$ we see that cooperation cannot evolve - i.e., no cooperative $\mathrm{ESS}_{\mathrm{N}}$ exists - unless the population size is greater than a critical population $\operatorname{size}^{\ddagger}$,

$$
N>N_{\min } \equiv n+\frac{n-1}{m} .
$$

Figure 2 illustrates this result for the particular NSG specified by the benefit function shown in figure 1. Put another way, for a given group size $n$, if the population size $N$ is too small then there is no cooperative $\mathrm{ESS}_{N}$, but if $N$ is sufficiently large then there is a (universal) cooperative $\mathrm{ESS}_{\mathrm{N}}$. For any given population size $N$, there are group sizes $n$ and benefit functions $B(\tau)$ that yield $N_{\min }>N$, so a qualitative difference between the evolutionary outcomes in finite and infinite populations can occur either for small or large population sizes.

ESS conditions in relation to group size $(n)$. Rearranging condition (11a) again, we can write

$$
n<n_{\mathrm{c}} \equiv \frac{m N+1}{m+1}= \begin{cases}\frac{1}{1-m(G-1)} & \text { if } m<1 /(G-1), \\ \infty & \text { otherwise }\end{cases}
$$

i.e., for cooperation to evolve, the group size $n$ must be less than $n_{\mathrm{c}}$, the maximum size of groups that support cooperation in a population of size $N$ (or a population divided into $G$

\footnotetext{
${ }^{\ddagger}$ Condition (c) in the definition of the NSG (§5.1) implies that $m>0$, so $N_{\min }$ is always well-defined in (13).
} 


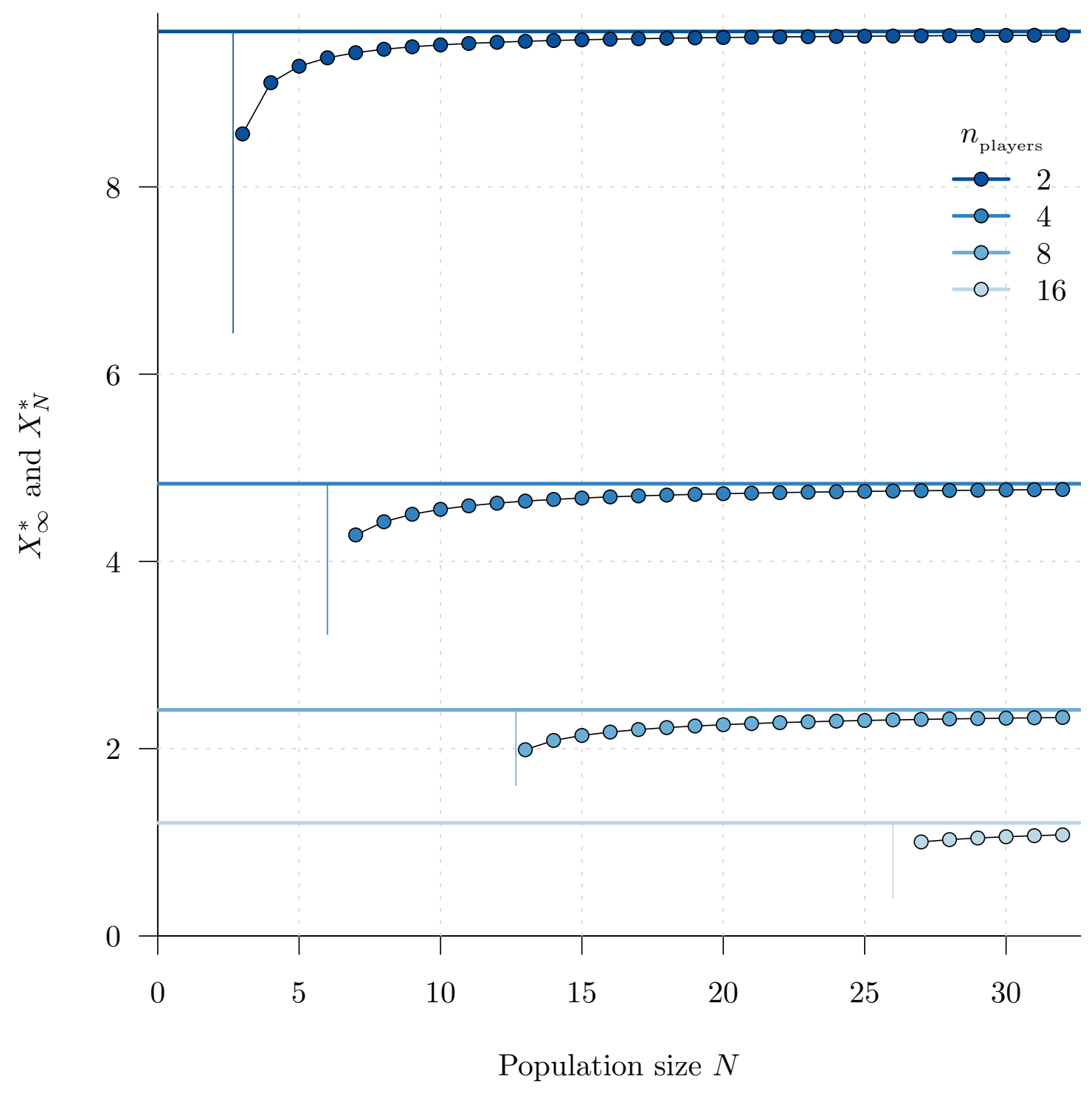

Figure 2: Evolutionarily stable strategies in the natural snowdrift game (Methods §5.1, with the sigmoidal benefit function shown in figure 1). For several group sizes $(n)$, the infinite population ESS $\left(X_{\infty}^{*}\right.$, equation (4)) is shown as a horizontal line, and the finite population $\operatorname{ESS}_{\mathrm{N}}\left(X_{N}^{*}\right)$ is shown with dots as a function of population size $N$. The vertical line segments indicate the critical population size threshold $\left(N_{\min }\right.$, inequality (13)). A cooperative $\mathrm{ESS}_{\mathrm{N}}$ exists if and only if $N>N_{\min }$. 
groups $^{\S}$ ). Multiplying inequality (14) by $G$ and rearranging, we obtain

$$
N<N_{\max } \equiv \begin{cases}\frac{G}{1-m(G-1)} & \text { if } m<1 /(G-1) \\ \infty & \text { otherwise }\end{cases}
$$

i.e., if the number of groups is fixed (and smaller than $1+1 / m$ ) then in order for a cooperative ESS $_{\mathrm{N}}$ to exist, the population size must be less than the threshold in inequality (15), as illustrated in figure 3.

Lack of $\mathbf{E S S}_{\mathbf{N}}$ for any population size. It is even possible that there is a cooperative ESS if the population is infinite, but no cooperative $\mathrm{ESS}_{\mathrm{N}}$ for any finite population size $N$. This is easy to verify for an NSG as follows. As noted above, an NSG always has an infinite-population cooperative ESS (4). An $\mathrm{ESS}_{\mathrm{N}}$ exists if and only if inequality (11a) [or inequality (14) or inequality (12)] is satisfied. Rearranging inequality (12) [or equation (10)], we can write, equivalently,

$$
m>m_{\mathrm{c}} \equiv \frac{1-(G / N)}{G-1}
$$

i.e., there is a cooperative $\mathrm{ESS}_{\mathrm{N}}$ if and only if the maximum marginal fitness $m$ exceeds the threshold $m_{\mathrm{c}}$ (exactly the same threshold that appears in equation (10), but expressed here in terms of $G$ rather than $n$ ). Suppose now that the population is divided into a given number of groups, $G \geq 2$. There must be at least two individuals in each group, so $N \geq 2 G$ and hence $G / N \leq 1 / 2$. Consequently, for any possible population size $N$, we have

$$
m_{\mathrm{c}} \geq \frac{1}{2(G-1)} .
$$

Therefore, if the benefit function is such that

$$
m<\frac{1}{2(G-1)}
$$

then no cooperative $\mathrm{ESS}_{\mathrm{N}}$ exists, no matter how large the population size $N$. Yet, when the game defined by the same cost and benefit functions is played in an infinite population, a cooperative ESS exists (regardless of the group size $n$ ). Given $G$, in the example of the NSG defined using equation (23), it is easy to satisfy inequality (18) since the only constraint on $m$ is that it must be positive.

Above, we have considered populations divided into a given number of groups. Alternatively, we could consider groups of a given size $(n)$, and ask whether it is possible for a public goods game to have a cooperative ESS if the population is infinite but no cooperative $\mathrm{ESS}_{\mathrm{N}}$ for any finite population size. As we show elsewhere, NSGs do not have this property, but there are snowdrift games that do have it (41).

Confirmation with both selection and mutation. Lastly, in figure 4 we complement our rigorous analyses with individual-based simulations of finite populations in which individuals undergo both selection and mutation (see appendix 5.2 for details). Simulations such

\footnotetext{
${ }^{\S}$ Note that $n_{\mathrm{c}}$ is always finite for a given population size, but when the number of groups $G$ is fixed and larger than $1+1 / m$, then there is an $\operatorname{ESS}_{\mathrm{N}}$ for any number of players $n$.
} 


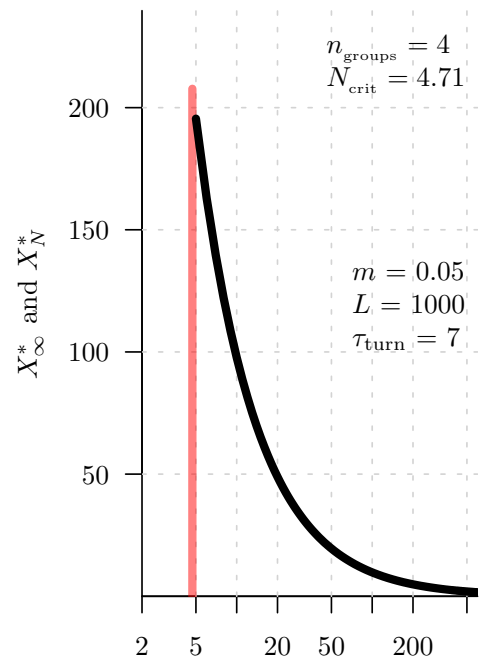

Population size $N$

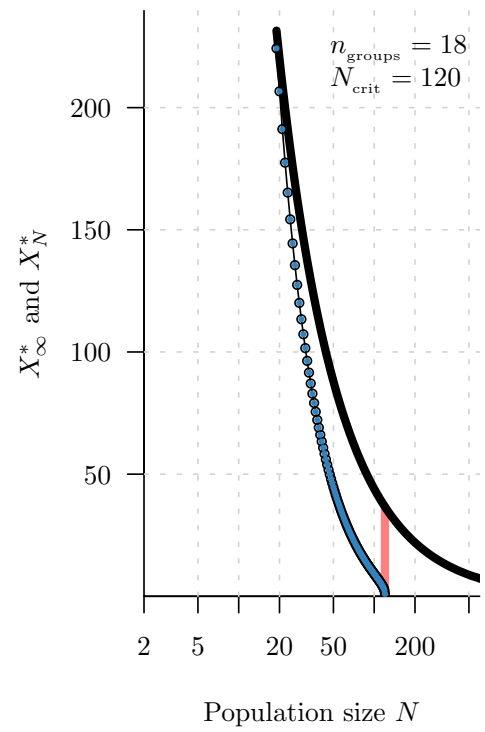

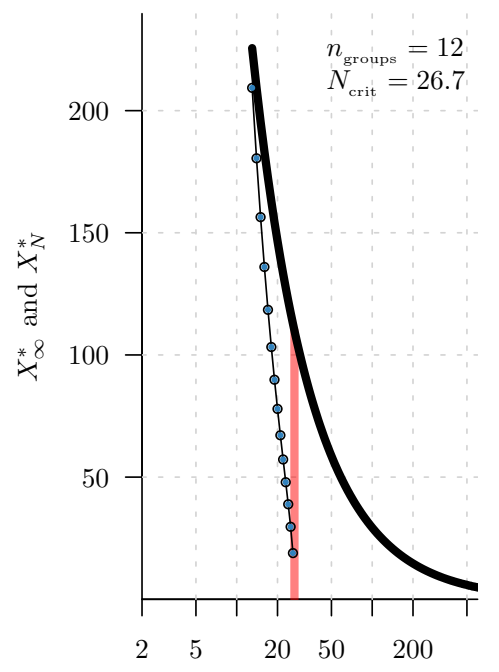

Population size $N$

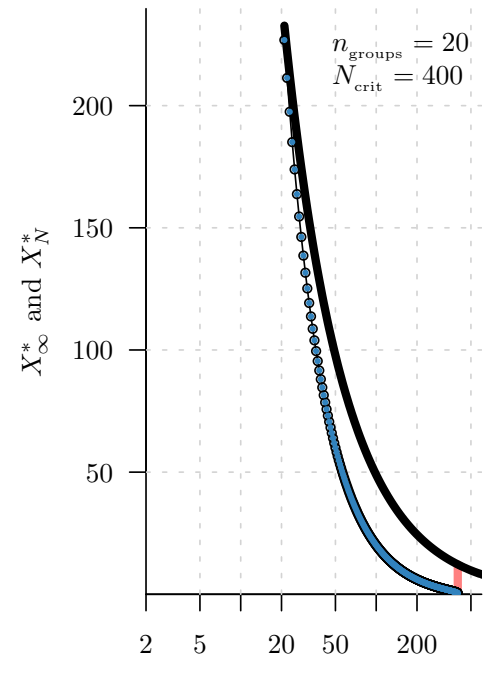

Population size $N$

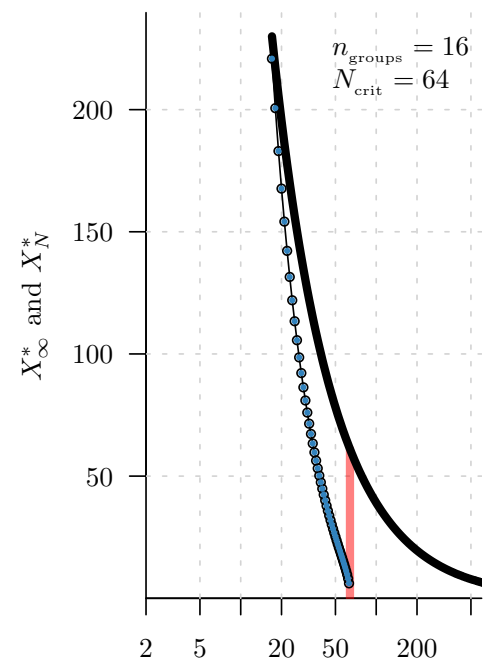

Population size $N$

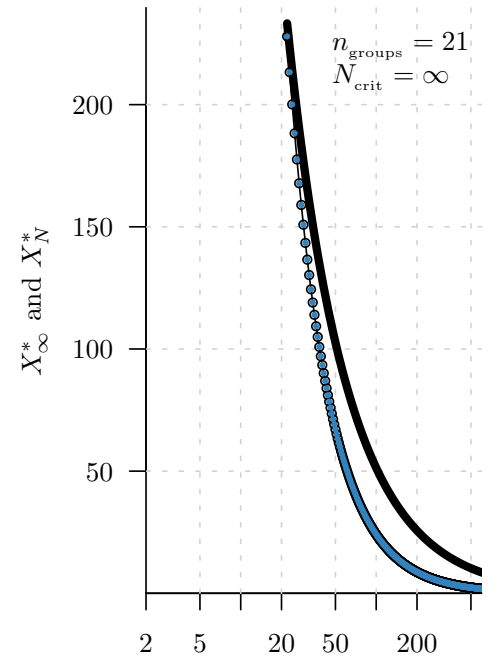

Population size $N$

Figure 3: Evolutionarily stable strategies in the natural snowdrift game (Methods §5.1), with the sigmoidal benefit function $B(\tau)$ given in Methods equation (23); parameter values are $L=1000$, $k=1, m=0.05, \tau_{\text {turn }}=7$. For several numbers of groups $(G)$, the infinite population ESS $\left(X_{\infty}^{*}\right.$, equation (4)) is shown as a curve, and the finite population $\operatorname{ESS}_{N}\left(X_{N}^{*}\right)$ is shown with dots as a function of population size $N$. For each number of groups, the minimum population considered is $N=G+1$. The vertical line segments indicate the critical population size threshold $\left(N_{\max }\right.$, inequality (15)), below which a cooperative $\mathrm{ESS}_{\mathrm{N}}$ exists (in contrast to the situation in which the group size $n$ is fixed and an $\mathrm{ESS}_{\mathrm{N}}$ exists only above a critical population size; $c f$. figure 2). 
as these confirm that rigorous game-theoretical analyses - which are based on selection acting with only two types in the population - correctly predict evolutionary outcomes in realistic populations in which each individual can, in principle, be playing a different strategy.

\section{Discussion}

We have seen that the evolutionary dynamics of the class of natural snowdrift games (NSGs, defined in Methods \$5.1) are different when played in finite vs. infinite populations. Since all real populations are finite, it is important to understand how inferences based on infinitepopulation analyses of the multi-player snowdrift game (e.g., $(3,30,42))$ might be affected. More generally, under what circumstances are infinite-population analyses of the evolution of cooperation likely to lead to invalid inferences about real populations?

We have shown that there are games for which it is possible that cooperation can evolve in an infinite population but not in any finite population (no matter how large). This extreme possibility emphasizes that inferences drawn from infinite population analyses should always be regarded cautiously.

The infinite-population approximation is likely to predict incorrect evolutionary outcomes if the number of individuals playing the game (the group size, $n$ ) is substantial relative to the total population size $(N)$. Exactly what "substantial" means will depend on the game in question and the population size; we have specified this threshold precisely for NSGs in inequality (14). Evolutionary predictions derived from infinite population analyses can be incorrect for finite populations of any size (figure 2 and theorem 2). The origin of such erroneous inferences is that finite groups (no matter how large) are always negligible in size compared to an infinite underlying population, but not compared to a finite underlying population.

Intuition for how different predictions arise in finite and infinite populations can be developed by considering a thought experiment in which the population (of size $N$ ) is simultaneously divided into $G$ groups that play the game. If a single mutant invades the resident population, the probability that a randomly chosen group contains the mutant is $1 / G$. If the population size were then increased by adding more and more groups of the same size $(G \rightarrow \infty$, keeping $n$ fixed), then the effect of the mutant on the residents would be "infinitely diluted" (the mutant would have a negligible effect on residents' fitnesses as $N \rightarrow \infty$ ). If, instead, the population size were increased by adding individuals to the existing groups (without increasing the number of groups) then the probability that a randomly selected group contains the mutant would not change; however, in this version of the thought experiment, the limit $N \rightarrow \infty$ entails the size of each group also becoming infinitely large.

Adaptive dynamics, which has been extensively used in the study of evolutionary dynamics [e.g., $(3,42,43)$, as well as (44) and references therein], relies on an infinite-population approximation (8). Previous work has presented reasonable arguments to justify this approximation (e.g., (35)) and reported general agreement between adaptive dynamics and stochastic simulations of finite populations (see (45) for a review). In addition, specific agreement has been noted (15) between the finite- and infinite-population evolutionary dynamics of the multi-player snowdrift game with discrete strategies. These results appear to contrast those presented here, though (15) did observe that defectors prevail when the group 


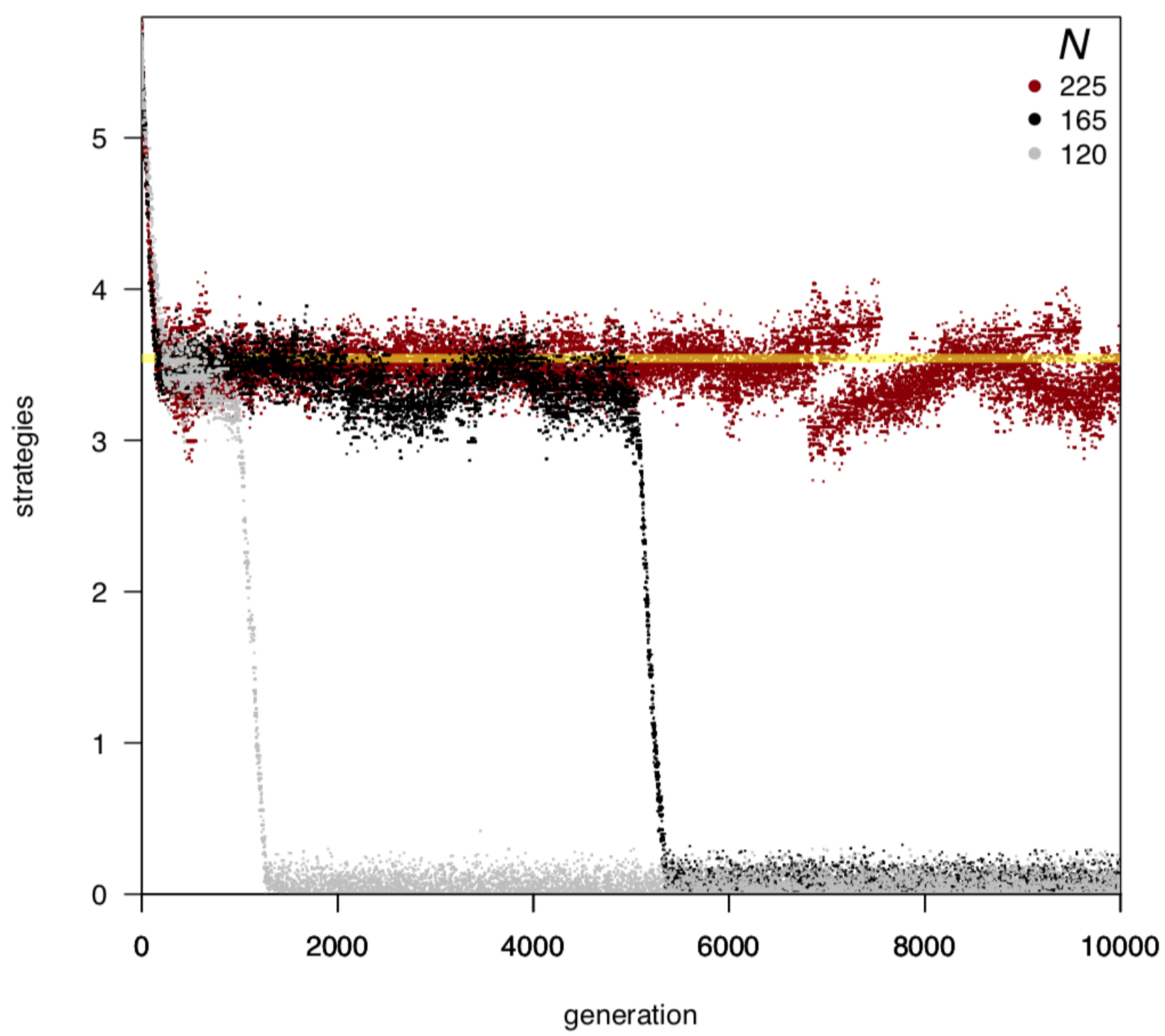

Figure 4: Individual-based simulations (details in appendix 5.2) of populations playing an NSG with cost and benefit functions as in figure 1 and group size $n=15$, for population sizes $N=225$ (red), 165 (black) and 120 (grey). The horizontal axis is the number of generations elapsed, and the vertical axis is the strategy (contribution level) of each individual in the population. The strategies present in the population in each generation are plotted on a vertical line intersecting the horizontal axis at the corresponding point. For $N=120$, defecting is the unique, globally convergently stable $\mathrm{ESS}_{\mathrm{N}}$; for $N>155$, a cooperative $\mathrm{ESS}_{\mathrm{N}}$ is predicted at $X^{*}=3.54$ (marked with a horizontal yellow line). The ESS for an infinite population playing this game is $X_{\infty}^{*}=3.56$. Note in these simulations, the mutation rate is high enough that populations contain more than two strategies at any given generation (in contrast to our rigorous mathematical analysis of dimorphic populations). 
size approaches the population size (even in situations in which cooperators and defectors can coexist in an infinite population). In other work, there has been a focus on situations in which the group size is much smaller than the population size, which reduces the chance of discovering discrepancies between finite and infinite population evolutionary predictions.

Our analysis of the class of natural snowdrift games is rigorous (theorems 1 and 2), and our conditions for existence of a cooperative $\mathrm{ESS}_{\mathrm{N}}$ are universal (in the sense of being entirely independent of the selection process). Broadly, our results indicate that approximating large populations by infinite ones may generate misleading conclusions. In particular, inferences based on adaptive dynamics are not necessarily applicable to real (finite) populations. There is a general need to reevaluate the theoretical justification for approximating large populations by infinite ones, and to derive clear conditions for when such approximations are useful.

\section{Methods}

\subsection{The natural snowdrift game (NSG)}

This biologically motivated version of the continuous snowdrift game (§2) was introduced in (30). We consider a population of individuals that are identical except (possibly) with respect to the strategy (contribution level) adopted when playing the snowdrift game. In particular, there is no age, spatial, social or other structure in the population. Evolution affects only the contribution levels of individuals, so at any time the population is completely characterized by the set of strategies present in the population and the numbers of individuals (or population proportions) playing each strategy. An individual's fitness is determined entirely by its payoff from the continuous snowdrift game played in groups of $n$ individuals. We say that this population plays a natural snowdrift game (NSG) if, in addition, the cost and benefit functions have the following properties (which are satisfied by the example shown in figure 1):

(a) The cost to the focal individual of a contribution $x$ is measured in units of its impact on this individual's fitness, that is,

$$
C(x)=x .
$$

Thus, the focal individual's fitness is

$$
W(x, \tau)=B(\tau)-x,
$$

where $\tau$ is the total contribution in the focal individual's group.

(b) The benefit $B(\tau)$ is a smooth function of the total contribution $\tau$ (more precisely, $B^{\prime \prime}(\tau)$ exists for all $\tau \geq 0)$.

(c) There exist total contribution levels $\tau_{\min }$ and $\tau_{\max }\left(0 \leq \tau_{\min }<\tau_{\max }\right)$ such that $B(\tau)-\tau$ decreases for $\tau<\tau_{\min }$ and $\tau>\tau_{\max }$ and increases for $\tau_{\min }<\tau<\tau_{\max }$. Consequently, given condition (a), if only one member of a group contributes anything then that individual's fitness [take $x=\tau$ in equation (20)] is locally minimized (maximized) if its contribution is $x=\tau_{\min }\left(\tau_{\max }\right)$. 
(d) There is a net fitness cost to an an individual who contributes $\tau_{\max }$ when all other group members contribute nothing,

$$
B\left(\tau_{\max }\right)<C\left(\tau_{\max }\right),
$$

but there is a net incremental fitness benefit for contributing $\tau_{\max } / n$ if other group members contribute that amount,

$$
B\left(\tau_{\max }\right)-B\left((n-1) \frac{\tau_{\max }}{n}\right)>C\left(\frac{\tau_{\max }}{n}\right) .
$$

In an infinite population, condition (c) implies that $\tau_{\max } / n$ and 0 are the only local ESSs (30). Adding condition (d) guarantees that they are both global ESSs [0 via and condition (21) and $\tau_{\max } / n$ via condition (22); see (30)].

\subsubsection{Benefit function used for numerical examples}

For the purpose of making example graphs and running simulations, we have used sigmoidal benefit functions. The biological motivation for this is that one would expect a nonlinear increase in the ease of passing the barrier as more snow is cleared, but eventually there can be no further benefit from additional work because all the snow has been cleared.

Specifically, for any integer $k>0$ and real numbers $m>0, L>0$ and $\tau_{\text {turn }} \geq 0$, consider the benefit function

$$
B(\tau)=L \operatorname{erf}_{2 k}\left((m+1) \frac{\Gamma(1 /(2 k))}{2 k L}\left(\tau-\tau_{\text {turn }}\right)\right), \quad \tau \geq 0,
$$

where $\operatorname{erf}_{\ell}(x)$ is the generalized error function (46) of order $\ell$,

$$
\operatorname{erf}_{\ell}(x)=\frac{\ell}{\Gamma(1 / \ell)} \int_{0}^{x} e^{-t^{\ell}} \mathrm{d} t
$$

and $\Gamma(x)$ is the gamma function [equation (50a)]. We analyze this flexible class of sigmoidal benefit functions in appendix $\mathrm{B}$, where we show that the parameters $L$ and $\tau_{\text {turn }}$ are the horizontal asymptote and the inflection point, respectively, $k$ controls the "width" of the sigmoid", and $m+1$ is the maximal marginal benefit (so that $m$ is the maximal marginal fitness that results from this functional form, justifying our notation).

Figure 1 shows the benefit function (23) for particular values of $k, m, L$ and $\tau_{\text {turn }}$, together with the corresponding fitness function (20) that results if residents defect, or - in groups of two individuals - if residents play the infinite population ESS [equation (4)]. Based on equation (23), in appendix B we derive explicit formulae for $\tau_{\min }, \tau_{\max }$, and $X_{\infty}^{*}$ and $X_{N}^{*}$ (in terms of $m, L, \tau_{\text {turn }}$ and $k$ ).

The class of sigmoids based on generalized error functions is much more flexible than the more common "logistic" sigmoid used by $(30,42)$ (which is based on shifting, and horizontally and vertically stretching, the hyperbolic tangent function, $\tanh (x)$ ). Whereas the maximum slope, horizontal asymptote and position of the inflection point uniquely determine the "width" of a logistic sigmoid, the generalized error function allows the width to be set independently via the parameter $k$ [see equation (60)].

"More precisely, for a given maximal marginal fitness $(m)$ and horizontal asymptote $(L), k$ controls the distance between the benefit function's inflection point $\left(\tau_{\text {turn }}\right)$ and the total contribution at which the marginal benefit is half of its maximum. 


\subsection{Individual-based simulations}

The three individual-based simulations shown in figure 4 [for population sizes $N=120$ (grey), 165 (black) and 250 (red)] were run using algorithm 1, which we implemented in an $\mathrm{R}$ (47) package. In the following description, we denote the normal distribution truncated to the interval $(l, u)$ by TruncNormal $(\mu, \sigma, l, u)$. It is a assumed that values of the following parameters have been set:

- Parameters $\left(k, m, L\right.$ and $\left.\tau_{\text {turn }}\right)$ of the benefit function (23).

- Group size $(n)$ and population size $(N)$, such that $G=N / n$ is an integer.

- Number of repetitions of the NSG between reproductive events $\left(n_{\text {reps }}\right)$.

- Maximum number of generations to evolve (nGen).

- Upper bound for contribution level $\left(x_{\max }\right)$.

- Mean $\left(\mu_{x}\right)$ and standard deviation $\left(\sigma_{x}\right)$ of an underlying Normal $\left(\mu_{x}, \sigma_{x}\right)$ distribution of strategies; the initial strategies $\left(x_{i}, i=1, \ldots, N\right)$ are to be sampled from TruncNormal $\left(\mu_{x}, \sigma_{x}, 0, x_{\max }\right)$.

- Mutation probability $\left(p_{\text {mut }}\right)$ per individual per generation.

- Standard deviation $(\sigma)$ of an underlying $\operatorname{Normal}(0, \sigma)$ distribution of the strategy changes caused by mutations, and upper and lower bounds on mutation sizes, $(l, u)$; when an individual playing strategy $x$ mutates, its new strategy is sampled from TruncNormal $\left(x, \sigma, \max \{0, x-l\}, \min \left\{x_{\max }, x+u\right\}\right)$, so that the mutation is within the interval $[l, u]$ and the mutated strategy is in $\left[0, x_{\max }\right]$.

\section{Acknowledgments}

DE was supported by the Natural Sciences and Engineering Research Council of Canada (NSERC). CM was supported by the Ontario Trillium Foundation, the United States Defense Advanced Research Project Agency NGS2 program (grant no. D17AC00005), and the Army Research Office (grant no. W911NF1810325). We are grateful to Sigal Balshine, Ben Bolker, Michael Doebeli, Jonathan Dushoff, Gil Henriques, Paul Higgs and Rufus Johnstone for valuable discussions and comments.

\section{References}

[1] Lehmann L. The stationary distribution of a continuously varying strategy in a class-structured population under mutation-selection-drift balance. Journal of evolutionary biology. 2012;25(4):770-787. Available from: https://doi.org/10.1111/j. $1420-9101.2012 .02472 . x$.

[2] Geritz SAH, Kisdi É, Meszéna G, Metz JAJ. Evolutionarily singular strategies and the adaptive growth and branching of the evolutionary tree. Evolutionary Ecology. 1998 Jan;12(1):35-57. Available from: https://doi.org/10.1023/A:1006554906681. 


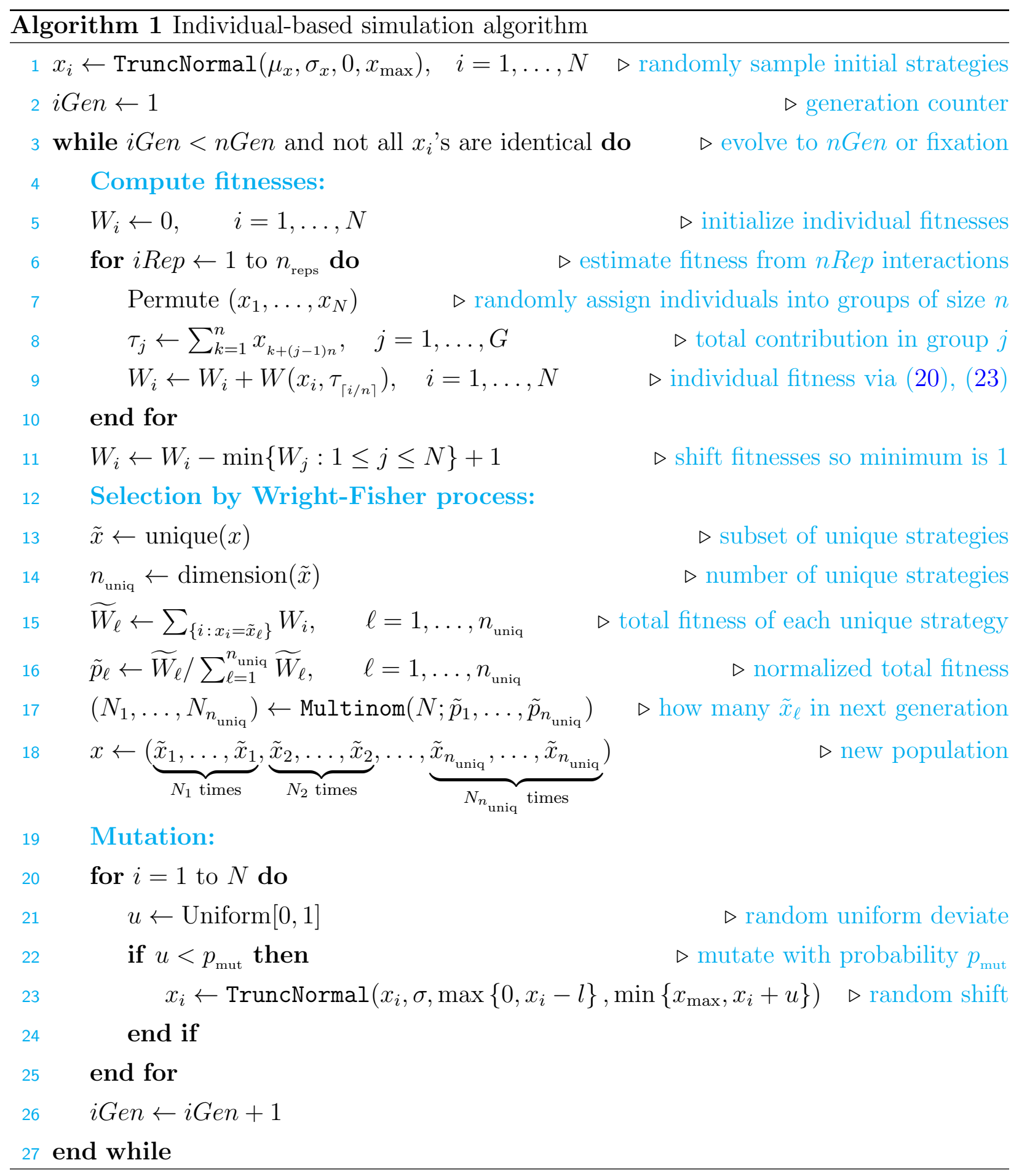


[3] Doebeli M, Hauert C, Killingback T. The evolutionary origin of cooperators and defectors. Science. 2004;306(5697):859-862. Available from: https://doi.org/10.1126/ science.1101456.

[4] Zheng DF, Yin H, Chan CH, Hui P. Cooperative behavior in a model of evolutionary snowdrift games with N-person interactions. EPL (Europhysics Letters). 2007;80(1):18002. Available from: https://doi.org/10.1209/0295-5075/80/18002.

[5] Brown JS, Vincent TL. Evolution of cooperation with shared costs and benefits. Proceedings of the Royal Society B. 2008 Sep;275(1646):1985-1994. Available from: http://dx.doi.org/10.1098/rspb.2007.1685.

[6] Brännström A, Gross T, Blasius B, Dieckmann U. Consequences of fluctuating group size for the evolution of cooperation. Journal of Mathematical Biology. 2010 Oct;63(2):263-281. Available from: http://dx.doi.org/10.1007/ s00285-010-0367-3.

[7] Deng K, Chu T. Adaptive Evolution of Cooperation through Darwinian Dynamics in Public Goods Games. PLoS ONE. 2011 Oct;6(10):e25496. Available from: http: //dx.doi.org/10.1371/journal.pone.0025496.

[8] Metz JA, Geritz SA, Meszéna G, Jacobs FJ, Van Heerwaarden JS. Adaptive dynamics, a geometrical study of the consequences of nearly faithful reproduction. In: van Strien SJ, Verduyn Lunel S, editors. Stochastic and Spatial Structures of Dynamical Systems. Amsterdam: North-Holland: Proceedings of the Royal Dutch Academy of Science (KNAW Verhandelingen); 1996. p. 183-231.

[9] Nowak MA, Sasaki A, Taylor C, Fudenberg D. Emergence of cooperation and evolutionary stability in finite populations. Nature. 2004;428(6983):646-650. Available from: https://doi.org/10.1038/nature02414.

[10] Crawford VP. Nash equilibrium and evolutionary stability in large-and finite-population playing the field models. Journal of Theoretical Biology. 1990;145(1):83-94. Available from: https://doi.org/10.1016/S0022-5193(05)80536-3.

[11] Wakano JY, Iwasa Y. Evolutionary branching in a finite population: deterministic branching versus stochastic branching. Genetics. 2012;p. 229-241. Available from: https://doi.org/10.1534/genetics.112.144980.

[12] Hauert C, Traulsen A, née Brandt HDS, Nowak MA, Sigmund K. Public goods with punishment and abstaining in finite and infinite populations. Biological Theory. 2008;3(2):114-122. Available from: https://doi.org/10.1162/biot.2008.3.2.114.

[13] Fogel GB, Andrews PC, Fogel DB. On the instability of evolutionary stable strategies in small populations. Ecological Modelling. 1998;109(3):283-294. Available from: https: //doi.org/10.1016/S0304-3800(98)00068-4.

[14] Imhof LA, Nowak MA. Evolutionary game dynamics in a Wright-Fisher process. Journal of Mathematical Biology. 2006;52(5):667-681. Available from: https://doi.org/10. 1007/s00285-005-0369-8. 
[15] Souza MO, Pacheco JM, Santos FC. Evolution of cooperation under N-person snowdrift games. Journal of Theoretical Biology. 2009;260(4):581-588. Available from: https: //doi.org/10.1016/j.jtbi.2009.07.010.

[16] Antal T, Nowak MA, Traulsen A. Strategy abundance in $2 \times 2$ games for arbitrary mutation rates. Journal of Theoretical Biology. 2009;257(2):340-344. Available from: https://doi.org/10.1016/j.jtbi.2008.11.023.

[17] Traulsen A, Claussen JC, Hauert C. Coevolutionary dynamics in large, but finite populations. Physical Review E. 2006;74(1):011901. Available from: https://doi .org/10. 1103/PhysRevE.74.011901.

[18] Lessard S. Long-term stability from fixation probabilities in finite populations: new perspectives for ESS theory. Theoretical population Biology. 2005;68(1):19-27. Available from: https://doi.org/10.1016/j.tpb.2005.04.001.

[19] Wakano JY, 'n L. Evolutionary and convergence stability for continuous phenotypes in finite populations derived from two-allele models. Journal of Theoretical Biology. 2012;310:206-215. Available from: https://doi.org/10.1016/j.jtbi.2012.06.036.

[20] Li K, Cong R, Wu T, Wang L. Social exclusion in finite populations. Physical Review E. 2015 Apr;91(4). Available from: http://dx.doi.org/10.1103/PhysRevE.91.042810.

[21] Chen X, Szolnoki A, Perc M, Wang L. Impact of generalized benefit functions on the evolution of cooperation in spatial public goods games with continuous strategies. Physical Review E. 2012 Jun;85(6). Available from: http://dx.doi.org/10.1103/ PhysRevE.85.066133.

[22] McGill BJ, Brown JS. Evolutionary Game Theory and Adaptive Dynamics of Continuous Traits. Annual Review of Ecology and Systematics. 2007 Dec;38(1):403-435. Available from: http://dx.doi.org/10.1146/annurev.ecolsys.36.091704.175517.

[23] Ewens WJ. Mathematical Population Genetics 1: Theoretical Introduction. vol. 27. Springer Science \& Business Media; 2012.

[24] Hartl DL, Clark AG. Principles of Population Genetics. Sinauer Associates; 2007.

[25] Ito K, Ohtsuki H, Yamauchi A. Relationship between aggregation of rewards and the possibility of polymorphism in continuous snowdrift games. Journal of Theoretical Biology. 2015 May;372:47-53. Available from: http://dx.doi.org/10.1016/j.jtbi . 2015.02 .015$.

[26] Sasaki T, Okada I. Cheating is evolutionarily assimilated with cooperation in the continuous snowdrift game. Biosystems. 2015 May;131:51-59. Available from: http: //dx.doi.org/10.1016/j.biosystems.2015.04.002.

[27] McNamara JM, Barta Z, Fromhage L, Houston AI. The coevolution of choosiness and cooperation. Nature. 2008 Jan;451(7175):189-192. Available from: http://dx.doi . org/10.1038/nature06455. 
[28] Zhong LX, Qiu T, Xu JR. Heterogeneity Improves Cooperation in Continuous Snowdrift Game. Chinese Physics Letters. 2008 May;25(6):2315-2318. Available from: http: //dx.doi.org/10.1088/0256-307X/25/6/107.

[29] Zhang Y, Fu F, Wu T, Xie G, Wang L. A tale of two contribution mechanisms for nonlinear public goods. Scientific Reports. 2013 Jun;3. Available from: http://dx. doi.org/10.1038/srep02021.

[30] Molina C, Earn DJD. Evolutionary stability in continuous nonlinear public goods games. Journal of Mathematical Biology. 2017;74(1-2):499-529. Available from: https://doi. org/10.1007/s00285-016-1017-1.

[31] Molina C, Earn DJD. On selection in finite populations. Journal of Mathematical Biology. 2018 Feb;76(3):645-678. Available from: https://doi.org/10.1007/ s00285-017-1151-4.

[32] Traulsen A, Claussen JC, Hauert C. Coevolutionary Dynamics: From Finite to Infinite Populations. Phys Rev Lett. 2005 Dec;95:238701. Available from: https://link.aps . org/doi/10.1103/PhysRevLett.95.238701.

[33] Maynard Smith J. Evolution and the Theory of Games. Cambridge University Press; 1982.

[34] Eshel I. On the changing concept of evolutionary population stability as a reflection of a changing point of view in the quantitative theory of evolution. Journal of Mathematical Biology. 1996;34(5-6):485-510.

[35] Dieckmann U, Law R. The dynamical theory of coevolution: a derivation from stochastic ecological processes. Journal of Mathematical Biology. 1996 May;34(5):579-612. Available from: https://doi.org/10.1007/BF02409751.

[36] Brännström A, Johansson J, von Festenberg N. The Hitchhiker's Guide to Adaptive Dynamics. Games. 2013;4(3):304-328. Available from: https://doi.org/10.3390/ g4030304.

[37] Molina C. The good, the finite, and the infinite [PhD dissertation]. McMaster University. Hamilton, ON, Canada; 2016. Available from: http://hdl. handle.net/11375/18951.

[38] Moran PAP. The statistical processes of evolutionary theory. Clarendon Press; 1962.

[39] Nowak MA. Evolutionary dynamics: Exploring the equations of life. Harvard University Press; 2006.

[40] Der R, Epstein CL, Plotkin JB. Generalized population models and the nature of genetic drift. Theoretical Population Biology. 2011;80(2):80 - 99. Available from: https: //doi.org/10.1016/j.tpb.2011.06.004.

[41] Molina C, Earn DJD. Selection may oppose invasion, yet favour fixation: consequences for evolutionary stability. bioRxiv. 2019;Available from: https://www.biorxiv.org/ content/early/2019/07/18/706879. 
[42] Cornforth DM, Sumpter DJ, Brown SP, Brännström A. Synergy and group size in microbial cooperation. The American Naturalist. 2012;180(3):296. Available from: https://doi .org/10.1086/667193.

[43] Killingback T, Doebeli M, Hauert C. Diversity of cooperation in the tragedy of the commons. Biological Theory. 2010;5:3-6. Available from: https://doi.org/10.1162/ BIOT_a_00019.

[44] Waxman D, Gavrilets S. 20 questions on adaptive dynamics. Journal of Evolutionary Biology. 2005;18(5):1139-1154. Available from: https://doi.org/10.1111/j. 1420-9101.2005.00948.x.

[45] Doebeli M. Adaptive dynamics: a framework for modeling the long-term evolutionary dynamics of quantitative traits. In: Svensson E, Calsbeek R, editors. The adaptive landscape in evolutionary biology. Oxford, U.K.: Oxford University Press; 2012. p. $227-242$.

[46] Yin L, Qi F. Some functional inequalities for generalized error function. J Comput Anal Appl. 2018;25:1366-1372.

[47] R Core Team. R: A Language and Environment for Statistical Computing. Vienna, Austria; 2016. Available from: https://www.R-project.org/.

[48] Ross SM. A First Course in Probability. Pearson Prentice Hall; 2010.

[49] Thomson BS, Bruckner JB, Bruckner AM. Elementary Real Analysis. 2nd ed. www.classicalrealanalysis.com.; 2008. Available from: https://books.google.com/ books?id=vA9d57GxCKgC. 


\section{SUPPORTING INFORMATION}

\section{A Proofs}

\section{A.1 Analysis of the natural snowdrift game (NSG; §5.1) in a finite population}

Our main results are stated in theorems 1 and $2(\S 3)$. Before developing the proofs in detail, it is useful to note that:

- $\tau_{\min }>0$ (where $\tau_{\min }$ is defined in assumption (c) of the definition of the NSG, $\S 5.1$ ). To see this, suppose that $\tau_{\min }=0$. Then assumption (c) implies that $B\left(\tau_{\max }\right) \geq \tau_{\max }$, contradicting assumption $(d)$.

- The benefit function $B(\tau)$ is twice-differentiable. This follows from assumption (b) in the definition of the NSG $(\S 5.1)$.

- $B^{\prime}\left(\tau_{\min }\right)=B^{\prime}\left(\tau_{\max }\right)=1, B^{\prime}(\tau)>1$ for $\tau_{\min }<\tau<\tau_{\max }$, and $B^{\prime}(\tau)<1$ otherwise [these properties of $B(\tau)$ follow from assumption (c)]. Consequently, $m>0$ and $B^{\prime \prime}\left(\tau_{\max }\right) \leq 0$.

\section{A.1.1 The mean fitness difference between mutants and residents}

Consider a population of $N$ individuals, comprised of $M_{\mathrm{p}}$ mutants who play $x$ and $N-M_{\mathrm{p}}$ residents who play $X$, and denote the proportion of mutants in the population by $\epsilon=M_{\mathrm{p}} / N$. Suppose that groups of $n$ individuals are randomly sampled from this population without replacement, which implies that the number of mutants in each such group is hypergeometrically distributed with parameters $N, M_{\mathrm{p}}$ and $n(37,48)$; thus, the probability of $k$ mutants occurring in a random sample of $n$ individuals is

$$
\operatorname{Pr}\left(M_{\mathrm{g}}=k\right)=\frac{\left(\begin{array}{c}
N-M_{\mathrm{p}} \\
n-k
\end{array}\right)\left(\begin{array}{c}
M_{\mathrm{p}} \\
k
\end{array}\right)}{\left(\begin{array}{c}
N \\
n
\end{array}\right)} .
$$

Suppose, moreover, that a focal individual is selected from the population by first sampling a group of $n$ individuals, and then selecting one of the members of this group. Lastly, suppose for simplicity that individual fitnesses are given by the payoffs from a single round of the NSG played in such randomly selected groups ${ }^{\#}$. We show elsewhere (37, eq. 4.61, p. 137) that the expected difference between the mutant and resident fitnesses is then

$$
\delta \bar{W}_{\epsilon}(x, X)=X-x+\sum_{k=0}^{n} \frac{\left(\begin{array}{c}
N-M_{\mathrm{p}} \\
n-k
\end{array}\right)\left(\begin{array}{c}
M_{\mathrm{p}} \\
k
\end{array}\right)}{\left(\begin{array}{c}
N-1 \\
n-1
\end{array}\right)}\left(\frac{k N-M_{\mathrm{p}} n}{M_{\mathrm{p}}\left(N-M_{\mathrm{p}}\right)}\right) B(k x+(n-k) X) .
$$

\#Equation (26) remains valid if individual fitnesses are obtained by averaging payoffs from an arbitrary (either fixed or random) number of rounds of the NSG, as long as groups are selected independently in each round. 
Differentiating equation (26) yields

$$
\begin{aligned}
\partial_{x} \delta \bar{W}_{\epsilon}(x, X) & =-1+\sum_{k=0}^{n} \frac{\left(\begin{array}{c}
N-M_{\mathrm{p}} \\
n-k
\end{array}\right)\left(\begin{array}{c}
M_{\mathrm{p}} \\
k
\end{array}\right)}{\left(\begin{array}{c}
N-1 \\
n-1
\end{array}\right)} \frac{k N-M_{\mathrm{p}} n}{M_{\mathrm{p}}\left(N-M_{\mathrm{p}}\right)} k B^{\prime}(k x+(n-k) X) \\
& =-1+\sum_{k=0}^{n} \frac{\left(\begin{array}{c}
N-M_{\mathrm{p}} \\
n-k
\end{array}\right)\left(\begin{array}{c}
M_{\mathrm{p}}-1 \\
k-1
\end{array}\right)}{\left(\begin{array}{c}
N-1 \\
n-1
\end{array}\right)} \cdot \frac{k N-M_{\mathrm{p}} n}{N-M_{\mathrm{p}}} B^{\prime}(k x+(n-k) X)
\end{aligned}
$$

Differentiating with respect to $x$ and setting $x=X$, we find (37, pp.138-139)

$$
\begin{aligned}
& \left.\partial_{x} \delta \bar{W}_{\epsilon}(x, X)\right|_{x=X}=-1+\frac{N-n}{N-1} B^{\prime}(n X), \\
& \left.\partial_{x}^{2} \delta \bar{W}_{\epsilon}(x, X)\right|_{x=X}=\frac{N-n}{N-1}\left(\frac{N-2 n}{N-2}+2 \frac{(n-1)}{N-2} N \epsilon\right) B^{\prime \prime}(n X) .
\end{aligned}
$$

From these expressions we see that

- $\left.\partial_{x} \delta \bar{W}_{\epsilon}(x, X)\right|_{x=X}$ is independent of $\epsilon$, and

- $\left.\partial_{x}^{2} \delta \bar{W}_{\epsilon}(x, X)\right|_{x=X}$ is linear in $\epsilon$.

We will exploit these facts below.

\section{A.1.2 Evolutionary and convergent stability of defection}

Lemma 3 (Evolutionary stability of defection). If the NSG (\$ 5.1) is played in a finite population then not contributing $(X=0)$ is a locally convergently stable $E S S_{N}$ for any selection process. Moreover, if the population and group sizes are the same $(N=n$, so the entire population plays the game together) then defecting is the unique $E S S_{N}$ and is globally evolutionarily and convergently stable.

Proof. $B^{\prime}(0)<1$ because $B(\tau)-\tau$ decreases for $0 \leq \tau<\tau_{\min }$, so using equation (28a),

$$
\left.\partial_{x} \delta \bar{W}_{\epsilon}(x, 0)\right|_{x=0}=\frac{N-n}{N-1} B^{\prime}(0)-1<0 .
$$

Because $\left.\partial_{x} \delta \bar{W}_{\epsilon}(x, X)\right|_{x=X}$ is continuous in $X$,

$$
\left.\partial_{x} \delta \bar{W}_{\epsilon}(x, X)\right|_{x=X}<0,
$$

for $X$ sufficiently small. From Theorem 4.3.9 in (37), it follows that $X=0$ (defection) is convergently stable, and selection opposes invasion of mutants contributing a sufficiently small but positive amount, $x>0$. To establish that $X=0$ is evolutionarily stable, observe that equation (29) implies that $\delta \bar{W}_{\epsilon}(x, 0)<0$ for sufficiently small $x$, so such mutants are selected against, regardless of their proportion $(\epsilon)$ in the population. Thus, corollary 5.4 of (31) implies that selection also opposes the fixation of such mutants.

Now suppose groups constitute the entire population, i.e., $N=n$. Then, for any resident strategy $X>0$ and any number of mutants $M_{\mathrm{p}} \in\{1,2, \ldots, N-1\}$, mutants contributing less than residents to the public good $(0 \leq x<X)$ have a higher payoff than residents; hence defection is the unique $\mathrm{ESS}_{\mathrm{N}}$ and is globally convergently stable. Defection is also globally evolutionarily stable because for any mutant strategy $x>0$ and any number of mutants $\left(M_{\mathrm{p}}<N\right)$, residents obtain a higher payoff than mutants (because they receive the same benefit without paying a cost). 


\section{A.1.3 Proof of theorem 1}

Inserting equation (28a) into the definition of an evolutionarily singular strategy (definition 4.3.5 of (37)) implies that cooperative singular strategies are characterized by equation (5). Any solution of equation (5) must satisfy $n X_{N}^{*} \in\left(\tau_{\min }, \tau_{\max }\right)$, because the right hand side of equation (5) is greater than 1 and, as noted above, if $\tau \notin\left(\tau_{\min }, \tau_{\max }\right)$ then $B^{\prime}(\tau) \leq 1$.

Necessary condition for $\mathbf{E S S}_{\mathbf{N}}$ : Suppose that $X$ solves equation (5) but $B^{\prime \prime}(n X)>$ 0 . Plugging equation (5) into equation (28a) gives $\left.\partial_{x} \delta \bar{W}_{\epsilon}(x, X)\right|_{x=X}=0$. Rearranging equation (28b), we have

$$
\left.\partial_{x}^{2} \delta \bar{W}_{\epsilon}(x, X)\right|_{x=X}=\frac{N-n}{N-1}\left(1+2 \frac{(n-1)}{N-2}(N \epsilon-1)\right) B^{\prime \prime}(n X)
$$

so $\left.\partial_{x}^{2} \delta \bar{W}_{\epsilon}(x, X)\right|_{x=X}$ is increasing in $\epsilon$ and positive for any $\epsilon \geq 1 / N$ (i.e., any mixed population). Thus, when mutants play $x$ sufficiently close to $X,\left.\partial_{x} \delta \bar{W}_{\epsilon}(x, X)\right|_{x=X}$ is negative for $x<X$ and positive for $x>X$; hence, since $\delta \bar{W}_{\epsilon}(X, X)=0$, we must have $\delta \bar{W}_{\epsilon}(x, X)>0$ for any $x$ that is near but not equal to $X$ (and this is true for any number of mutants $\left.M_{\mathrm{p}}=1, \ldots, N-1\right)$. Corollary 5.4 of (31) then implies that selection favours the fixation of such mutants, so $X$ is not an $\mathrm{ESS}_{\mathrm{N}}$, regardless of the selection process. Thus, if $X_{N}^{*}>0$ is an $\mathrm{ESS}_{\mathrm{N}}$ then it cannot be that $B^{\prime \prime}(n X)>0$, i.e., inequality (7) holds.

Sufficient condition for universal ESS $_{\mathbf{N}}$ : The sufficient condition for local universal evolutionary and convergent stability follows immediately from theorem 4.D.1 of (37) and equation (28).

ESS $_{\mathbf{N}} \mathbf{S}$ in large populations: Suppose that $B^{\prime \prime}\left(\tau_{\max }\right) \neq 0$ and consider the equation

$$
f(X, y)=B^{\prime}(n X)-y=0 .
$$

Noting that $f\left(\tau_{\max } / n, 1\right)=0$ and that

$$
\left.\partial_{X} f(X, y)\right|_{(X, y)=\left(\tau_{\max } / n, 1\right)}=B^{\prime \prime}\left(\tau_{\max }\right) \neq 0,
$$

from the implicit function theorem (49, Theorem 12.40), there exists a differentiable function $X(y)$ defined in a neighbourhood of $y=1$, such that $X(1)=\tau_{\max } / n$ and

$$
f(X(y), y)=y B^{\prime}(n X(y))-y=0 .
$$

Now suppose that the group size $n$ is either fixed, or varies with population size but satisfies

$$
\frac{n(N)}{N} \stackrel{N \rightarrow \infty}{\longrightarrow} 0 \text {. }
$$

If we define $y_{N}:=1+\frac{n-1}{N-n}$ then $y_{N} \rightarrow 1$, so for all sufficiently large population sizes $N$, equation (34) can be solved implicitly for $X_{N}^{*}:=X\left(y_{N}\right)$. Such $X_{N}^{*}$ then solve equation (5), and $X_{N}^{*} \stackrel{N \rightarrow \infty}{\longrightarrow} X_{\infty}^{*}$ because $X(y)$ is continuous. Recalling that $B^{\prime \prime}\left(\tau_{\max }\right) \leq 0$ and $B^{\prime \prime}\left(\tau_{\max }\right) \neq$ 0 by assumption, we have $B^{\prime \prime}\left(\tau_{\max }\right)<0$, so for sufficiently large $N, B^{\prime \prime}\left(n X_{N}^{*}\right)<0$. Theorem 4.D.1 of (37) then implies that for sufficiently large $N, X_{N}^{*}$ is a universal local $\mathrm{ESS}_{N}$ and is locally convergently stable. 


\section{A.1.4 Proof of theorem 2}

First, note that $X=0$ is always a locally convergently stable ESS $_{\mathrm{N}}$ (lemma 3 ). From Corollary 4.3 .8 of (37), selection opposes invasion of a cooperative resident strategy $X>0$ by sufficiently similar mutant strategies only if $X$ is singular, which (using equation (28a)) occurs iff $X$ satisfies

$$
B^{\prime}(n X)=1+\frac{n-1}{N-n} .
$$

Because $B^{\prime}(n X)>1$ only if $\frac{\tau_{\min }}{n}<X<\frac{\tau_{\max }}{n}$, if a cooperative $\mathrm{ESS}_{\mathrm{N}}$ exists then it must lie in this interval.

Case $\boldsymbol{m}>\boldsymbol{m}_{\mathbf{c}}$. Because $B^{\prime}\left(\tau_{\max }\right)=1$ and $B^{\prime}(n X)$ is a continuous function of $X$ on the interval $\left[\tau_{\min } / n, \tau_{\max } / n\right]$, it follows from the intermediate value theorem (49) that equation (5) has a solution in this interval. Let $\mathcal{S}$ be the set of singular strategies, i.e., solutions of equation (5),

$$
\mathcal{S}=\left\{X \mid B^{\prime}(n X)=1+\frac{n-1}{N-n}\right\} .
$$

Note that from theorem $1, \mathcal{S} \subset\left(\tau_{\min } / n, \tau_{\max } / n\right)$. Denote the largest solution of equation (35) by $X_{N}^{*}$, i.e.,

$$
X_{N}^{*}=\max \mathcal{S}
$$

(this maximum exists because the continuity of $B^{\prime}(n X)$ on a closed interval implies $\sup \mathcal{S} \in \mathcal{S})$.

Generically", $B^{\prime \prime}\left(n X_{N}^{*}\right) \neq 0$. We claim that $B^{\prime \prime}\left(n X_{N}^{*}\right)<0$. To see this, suppose, in order to derive a contradiction, that $B^{\prime \prime}\left(n X_{N}^{*}\right)>0$. Then, $B^{\prime}(n X)$ increases in a neighbourhood of $X_{N}^{*}$, so there exists $\tilde{X}$ such that $X_{N}^{*}<\tilde{X}<\tau_{\max } / n$ and

$$
B^{\prime}(n \tilde{X})>B^{\prime}\left(n X_{N}^{*}\right)=1+\frac{n-1}{N-n} .
$$

From the intermediate value theorem, there exists $X \in \mathcal{S}$ such that

$$
X>\tilde{X}>X_{N}^{*}=\max \mathcal{S},
$$

a contradiction.

Thus $B^{\prime \prime}\left(n X_{N}^{*}\right)<0$ and $C^{\prime \prime}(X)=0$, so theorems 4.D.1 and 4.3 .9 of (37) imply that $X_{N}^{*}$ is a local $\mathrm{ESS}_{\mathrm{N}}$ and is locally convergently stable.

Case $\boldsymbol{m}=\boldsymbol{m}_{\mathbf{c}}$. Suppose, in order to derive a contradiction, that $X>0$ is a cooperative $\mathrm{ESS}_{\mathrm{N}}$. From theorem 1, $X$ must solve equation (35) so, from the definition of $m_{\mathrm{c}}$ in equation (10),

$$
B^{\prime}(n X)=\frac{N-1}{N-n}=m_{\mathrm{c}}+1 .
$$

"We need to avoid the situation in which singular strategy $X_{N}^{*}$ is also an inflection point of $B(n x)$. This occurs when $n X_{N}^{*}$ is both a critical point and an inflection point of $B(x)-(N-1) x /(N-n)$, which is generically not the case. 
Suppose further that $\arg \max B^{\prime}(\tau)$ does not contain an interval (i.e., the marginal benefit $B^{\prime}$ is not maximal for an interval of total contributions $\tau$ ), which happens generically. Then, any total contribution in $\arg \max B^{\prime}(\tau)$ is a local maximum of $B^{\prime}(\tau)$. It follows that if $x<X$ and $x$ is sufficiently close to $X$, then

$$
B^{\prime}(x+(n-1) X)<\frac{N-1}{N-n},
$$

and therefore from equation (27),

$$
\begin{aligned}
\partial_{x} \delta \bar{W}_{\epsilon}(x, X) & =-1+\sum_{k=0}^{n} \frac{\left(\begin{array}{c}
N-M_{\mathrm{p}} \\
n-k
\end{array}\right)\left(\begin{array}{c}
M_{\mathrm{p}}-1 \\
k-1
\end{array}\right)}{\left(\begin{array}{c}
N-1 \\
n-1
\end{array}\right)} \cdot \frac{k N-M_{\mathrm{p}} n}{N-M_{\mathrm{p}}} B^{\prime}(k x+(n-k) X) \\
& <-1+\left(\sum_{k=0}^{n} \frac{\left(\begin{array}{c}
N-M_{\mathrm{p}} \\
n-k
\end{array}\right)\left(\begin{array}{c}
M_{\mathrm{p}}-1 \\
k-1
\end{array}\right)}{\left(\begin{array}{c}
N-1 \\
n-1
\end{array}\right)} \cdot \frac{k N-M_{\mathrm{p}} n}{N-M_{\mathrm{p}}}\right) \frac{N-1}{N-n},
\end{aligned}
$$

which, together with the identity (37, equation (4.63), p. 138),

$$
\sum_{k=0}^{n} \frac{\left(\begin{array}{c}
N-K \\
n-k
\end{array}\right)\left(\begin{array}{c}
K-1 \\
k-1
\end{array}\right)}{\left(\begin{array}{l}
N-1 \\
n-1
\end{array}\right)}\left(\frac{k N-K n}{N-K}\right)=\frac{N-n}{N-1},
$$

implies that $\partial_{x} \delta \bar{W}_{\epsilon}(x, X)<0$. Hence, similar to an argument in the proof of theorem 1 , since $\delta \bar{W}_{\epsilon}(X, X)=0$, we must have $\delta \bar{W}_{\epsilon}(x, X)>0$ for any $x$ that is slightly less than but not equal to $X$ (and this is true for any number of mutants $M_{\mathrm{p}}=1, \ldots, N-1$ ). Consequently, selection favours the invasion and replacement of $X$ by any such $x$, so $X$ is not evolutionarily stable.

To see that defection is globally evolutionarily stable, substitute $X=0$ in equation (27) to get

$$
\partial_{x} \delta \bar{W}_{\epsilon}(x, 0)=-1+\sum_{k=0}^{n} \frac{\left(\begin{array}{c}
N-M_{\mathrm{p}} \\
n-k
\end{array}\right)\left(\begin{array}{c}
M_{\mathrm{p}}-1 \\
k-1
\end{array}\right)}{\left(\begin{array}{c}
N-1 \\
n-1
\end{array}\right)} \cdot \frac{k N-M_{\mathrm{p}} n}{N-M_{\mathrm{p}}} B^{\prime}(k x) .
$$

Noting that for all $x>0, B^{\prime}(k x) \leq m_{\mathrm{c}}+1$, we have

$$
\partial_{x} \delta \bar{W}_{\epsilon}(x, 0) \leq-1+\left(\sum_{k=0}^{n} \frac{\left(\begin{array}{c}
N-M_{\mathrm{p}} \\
n-k
\end{array}\right)\left(\begin{array}{c}
M_{\mathrm{p}}-1 \\
k-1
\end{array}\right)}{\left(\begin{array}{l}
N-1 \\
n-1
\end{array}\right)} \cdot \frac{k N-M_{\mathrm{p}} n}{N-M_{\mathrm{p}}}\right)\left(m_{\mathrm{c}}+1\right)=0,
$$

where we have used equations (10) and (43) in the last equality. Thus, $\delta \bar{W}_{\epsilon}(x, 0)$ is non-decreasing in $x$. Moreover, if $x<\tau_{\min } / n$, then $B^{\prime}(k x)<1$ for all $k=0, \ldots, n$, so similarly, equations (43) and (44) imply that $\partial_{x} \delta \bar{W}_{\epsilon}(x, 0)<0$. Because $\delta \bar{W}_{\epsilon}(0,0)=0$, it follows that $\delta \bar{W}_{\epsilon}(x, 0)<0$ for all $x>0$ (regardless of the proportion of mutants in the population). Thus, from (31, corollary 5.4), when residents defect, selection opposes invasion and fixation of any mutants.

Case $\boldsymbol{m}<\boldsymbol{m}_{\mathbf{c}}$. In this case, equation (5) has no solution, and no cooperative $\mathrm{ESS}_{\mathrm{N}}$ exists. 
To see that defection $(X=0)$ is globally evolutionarily and convergently stable, observe first that $m<m_{\mathrm{c}}$ implies

$$
B^{\prime}(\tau)<1+\frac{n-1}{N-n}=\frac{N-1}{N-n}, \quad \text { for all } \quad \tau \geq 0 .
$$

Then, using equations (27), (46) and equation (4.63) on p.138 of (37), it follows that

$$
\begin{aligned}
\partial_{x} \delta \bar{W}_{\epsilon}(x, X) & <-1+\left(\sum_{k=0}^{n} \frac{\left(\begin{array}{c}
N-M_{\mathrm{p}} \\
n-k
\end{array}\right)\left(\begin{array}{c}
M_{\mathrm{p}}-1 \\
k-1
\end{array}\right)}{\left(\begin{array}{c}
N-1 \\
n-1
\end{array}\right)} \cdot \frac{k N-M_{\mathrm{p}} n}{N-M_{\mathrm{p}}}\right) \frac{N-1}{N-n} \\
& =-1+\left(\frac{N-n}{N-1}\right)\left(\frac{N-1}{N-n}\right)=0,
\end{aligned}
$$

so $\delta \bar{W}_{\epsilon}(x, X)$ decreases with $x \geq 0$ for any $X \geq 0$. Thus, from (31, corollary 5.4), defection $(X=0)$ is a globally evolutionarily and convergently stable strategy.

\section{B Analysis of the benefit function used for numerical examples}

In this appendix we define the class of sigmoidal benefit functions that we have used to illustrate our results, and derive a variety of analytical formulae that we have found useful when working with these functions.

\section{B.1 Sigmoids using generalized error functions}

For any integer $k>0$ and real $m>0, L>0$ and $\tau_{\text {turn }} \geq 0$, consider the benefit function

$$
B(\tau)=L \operatorname{erf}_{2 k}\left((m+1) \frac{\Gamma(1 /(2 k))}{2 k L}\left(\tau-\tau_{\text {turn }}\right)\right), \quad \tau \geq 0,
$$

where $\operatorname{erf}_{\ell}(x)$ is the generalized error function of order $\ell$,

$$
\operatorname{erf}_{\ell}(x)=\frac{\ell}{\Gamma(1 / \ell)} \int_{0}^{x} e^{-t^{\ell}} \mathrm{d} t
$$

This class of functions generalizes the the error function, erf, which is recovered for $\ell=2$ or, equivalently, $k=1$; see $\S$ B.2.

Expressing generalized error functions using gamma functions: It is sometimes convenient to express $\operatorname{erf}_{\ell}$ in terms of gamma functions. For $x>0$, the transformation $z=t^{\ell}\left(t=z^{1 / \ell}\right.$ and $\left.\mathrm{d} t=z^{\frac{1}{\ell}-1} \mathrm{~d} z / \ell\right)$ gives

$$
\operatorname{erf}_{\ell}(x)=\frac{1}{\Gamma(1 / \ell)} \int_{0}^{x^{\ell}} z^{\frac{1}{\ell}-1} e^{-z} \mathrm{~d} z=\frac{1}{\Gamma(1 / \ell)}\left(\Gamma\left(\frac{1}{\ell}\right)-\Gamma\left(\frac{1}{\ell}, x^{\ell}\right)\right)
$$




\footnotetext{
** For any positive integer $k, \Gamma(k)=(k-1)$ !.
} of $x$.

it follows that fitness given the benefit function $B$ is $m$.

SO

are the gamma**, and upper incomplete gamma functions, respectively. Note that we are only interested in generalized error functions of even order $(\ell=2 k)$, which are odd functions

Parameter meanings: Because equation (49) implies

$$
\lim _{x \rightarrow \infty} \operatorname{erf}_{\ell}(x)=1,
$$

$$
\lim _{x \rightarrow \infty} B(x)=L .
$$

We show below that the inflection point of $B(47)$ is $\tau_{\text {turn }}$, and that the maximal marginal

From the integral definition of the generalized error function [equation (48)]

$$
\begin{aligned}
\frac{\operatorname{derf}_{\ell}(x)}{\mathrm{d} x} & =\frac{\ell}{\Gamma(1 / \ell)} e^{-x^{\ell}}, \\
\frac{\mathrm{d}^{2} \operatorname{erf}}{\ell}(x) & =-\frac{\ell}{\Gamma(1 / \ell)} \ell x^{\ell-1} e^{-x^{\ell}},
\end{aligned}
$$

$$
\begin{aligned}
B^{\prime}(\tau)= & \frac{\sqrt{\pi} L}{\Gamma(2 k) \Gamma(1 /(2 k))}(m+1) \frac{\Gamma(1 /(2 k))}{2 k L} \frac{(2 k) !}{\sqrt{\pi}} \exp \left(-\left[(m+1) \frac{\Gamma(1 /(2 k))}{2 k L}\left(\tau-\tau_{\text {turn }}\right)\right]^{2 k}\right) \\
= & (m+1) \exp \left(-\left[(m+1) \frac{\Gamma(1 /(2 k))}{2 k L}\left(\tau-\tau_{\text {turn }}\right)\right]^{2 k}\right) \\
B^{\prime \prime}(\tau)= & -2 k\left[(m+1) \frac{\Gamma(1 /(2 k))}{2 k L}\right]^{2 k}\left(\tau-\tau_{\text {turn }}\right)^{2 k-1} \\
& \times(m+1) \exp \left(-\left[(m+1) \frac{\Gamma(1 /(2 k))}{2 k L}\left(\tau-\tau_{\text {turn }}\right)\right]^{2 k}\right) \\
= & -2 k\left[(m+1) \frac{\Gamma(1 /(2 k))}{2 k L}\right]^{2 k}\left(\tau-\tau_{\text {turn }}\right)^{2 k-1} B^{\prime}(\tau)
\end{aligned}
$$


Consequently, $\tau_{\text {turn }}$ is the unique solution of $B^{\prime \prime}(\tau)=0$, and is thus the only inflection point. $B^{\prime}(\tau)$ is always positive, and hence $B(\tau)$ is monotonically increasing. However, $B^{\prime \prime}(\tau)>0$ for $\tau<\tau_{\text {turn }}$ and $B^{\prime \prime}(\tau)<0$ for $\tau>\tau_{\text {turn }}$, and hence

$$
\max _{\tau \geq 0} B^{\prime}(\tau)=B^{\prime}\left(\tau_{\text {turn }}\right)=m+1,
$$

so from equation (9), the maximal marginal fitness is

$$
\max _{\tau \geq 0}\left(\frac{\partial W}{\partial x}\right)=\max _{\tau \geq 0} B^{\prime}(\tau)-1=m .
$$

The minimizing and maximizing total goods: Since $B^{\prime}(\tau)$ is monotonic on each of the intervals, $\left(-\infty, \tau_{\text {turn }}\right)$ and $\left(\tau_{\text {turn }}, \infty\right)$ and $B^{\prime}(\tau)$ is even, for any $b \in B^{\prime}\left(\mathbb{R}_{\geq 0}\right)=(0, m+1]$, we can find two real values of $\tau$ for which $B^{\prime}(\tau)=b$ (although one of these values may be negative and therefore biologically irrelevant, because total contributions to the public good cannot be negative). To find these values of total contribution $\tau$, we set $B^{\prime}(\tau)=b$ in equation $(54 \mathrm{a})$, and get

$$
\begin{aligned}
\log \frac{m+1}{b} & =\left[(m+1) \frac{\Gamma(1 /(2 k))}{2 k L}\left(\tau-\tau_{\text {turn }}\right)\right]^{2 k}, \\
\tau & =\tau_{\text {turn }} \pm \frac{2 k L}{(m+1) \Gamma(1 /(2 k))} \sqrt[2 k]{\log \frac{m+1}{b}}
\end{aligned}
$$

To find $\tau_{\max }$ and $\tau_{\min }$, we substitute $b=B^{\prime}(\tau)=1$ in equation (58) and, noting that $B^{\prime \prime}(\tau)$ changes sign from positive to negative at $\tau_{\text {turn }}$, we have

$$
\begin{aligned}
\tau_{\text {min }} & =\tau_{\text {turn }}-\frac{2 k L}{(m+1) \Gamma(1 /(2 k))} \sqrt[2 k]{\log (m+1)}, \\
\tau_{\max } & =\tau_{\text {turn }}+\frac{2 k L}{(m+1) \Gamma(1 /(2 k))} \sqrt[2 k]{\log (m+1)},
\end{aligned}
$$

and the distance between the location of the fitness minimum and maximum is

$$
\Delta \tau=\tau_{\max }-\tau_{\min }=\frac{4 k L}{(m+1) \Gamma(1 /(2 k))} \sqrt[2 k]{\log (m+1)} .
$$

The infinite-population cooperative ESS: Equation (4) then gives

$$
X_{\infty}^{*}=\frac{\tau_{\max }}{n}=\frac{1}{n}\left(\tau_{\text {turn }}+\frac{2 k L}{(m+1) \Gamma(1 /(2 k))} \sqrt[2 k]{\log (m+1)}\right) .
$$

Using $B^{\prime}\left(\tau_{\max }\right)=1$ and equation (59b) in equation (54b), we have

$$
\begin{aligned}
b_{\text {curve }} & :=B^{\prime \prime}\left(\tau_{\max }\right)=B^{\prime \prime}\left(n X_{\infty}^{*}\right)=-2 k\left[\frac{(m+1) \Gamma(1 /(2 k))}{2 k L}\right]^{2 k}\left(\tau_{\max }-\tau_{\text {turn }}\right)^{2 k-1} \\
& =-2 k\left[\frac{(m+1) \Gamma(1 /(2 k))}{2 k L}\right]^{2 k}\left(\frac{2 k L}{(m+1) \Gamma(1 /(2 k))}\right)^{2 k-1}(\log (m+1))^{\frac{2 k-1}{2 k}} \\
& =-\Gamma\left(\frac{1}{2 k}\right) \frac{m+1}{L}(\log (m+1))^{1-\frac{1}{2 k}} .
\end{aligned}
$$


Using equation (47) and the fact that $\operatorname{erf}_{2 k}$ is odd,

$$
B\left(\tau_{\max }\right)-B\left(\tau_{\min }\right)=2 L \operatorname{erf}_{2 k}(\sqrt[2 k]{\log (m+1)}) .
$$

Singular and evolutionarily stable cooperative strategies in finite populations: In a finite population of size $N$, a singular strategy $X_{N}^{*}$ of the NSG is a solution of equation (5), that is,

$$
B^{\prime}\left(n X_{N}^{*}\right)=1+\frac{n-1}{N-n}=\frac{N-1}{N-n} .
$$

so equation (58) implies that at the ESS, the total contribution must be one of

$$
\tau=\tau_{\text {turn }} \pm \frac{2 k L}{(m+1) \Gamma(1 /(2 k))} \sqrt[2 k]{\log \left((m+1) \frac{N-n}{N-1}\right)} .
$$

There are therefore two singular strategies,

$$
X_{N \pm}^{*}=\frac{1}{n}\left(\tau_{\text {turn }} \pm \frac{2 k L}{(m+1) \Gamma(1 /(2 k))} \sqrt[2 k]{\log \left((m+1) \frac{N-n}{N-1}\right)}\right)
$$

Similarly to $\tau_{\min }$ and $\tau_{\max }, B^{\prime \prime}\left(n X_{N+}^{*}\right)>0$ and $B^{\prime \prime}\left(n X_{N_{-}}^{*}\right)<0$, so from theorem 1 , the unique $\mathrm{ESS}_{\mathrm{N}}$ is

$$
X_{N}^{*}=\frac{1}{n}\left(\tau_{\text {turn }}+\frac{2 k L}{(m+1) \Gamma(1 /(2 k))} \sqrt[2 k]{\log \left((m+1) \frac{N-n}{N-1}\right)}\right) .
$$

The curvature of the benefit function at the $\mathbf{E S S}_{\mathbf{N}}$ : Similar to equation (62), we have

$$
\begin{aligned}
B^{\prime \prime}\left(n X_{N}^{*}\right)= & -2 k\left[\frac{(m+1) \Gamma(1 /(2 k))}{2 k L}\right]^{2 k}\left(n X_{N}^{*}-\tau_{\text {turn }}\right)^{2 k-1} \frac{N-1}{N-n} \\
= & -2 k\left[\frac{(m+1) \Gamma(1 /(2 k))}{2 k L}\right]^{2 k}\left(\frac{2 k L}{(m+1) \Gamma(1 /(2 k))}\right)^{2 k-1} \\
& \times\left(\log \left((m+1) \frac{N-n}{N-1}\right)\right)^{\frac{2 k-1}{2 k}} \frac{N-1}{N-n} \\
= & -(m+1) \frac{N-1}{N-n} \frac{\Gamma(1 /(2 k))}{L} \\
& \times\left(\log \left((m+1) \frac{N-n}{N-1}\right)\right)^{\frac{2 k-1}{2 k}}
\end{aligned}
$$

Condition for the fitness difference having a minimum when a single mutant defects and residents play the ESS: To guarantee that when a single mutant invades a population playing the ESS, the fitness difference has both a minimum and a maximum (as a 
function of the mutant strategy), we need the mutant contribution that minimizes fitness to be positive (or equivalently, the contribution of the nonfocal individuals - all residents - must be less than the minimizing total good $\left.\tau_{\min }\right)$,

$$
\tau_{\min }-\frac{n-1}{n} \tau_{\max }>0 \text {. }
$$

Using equations (59b) and (60), this is equivalent to $\tau_{\max }>n \Delta \tau$, or

$$
\begin{aligned}
n<\frac{\tau_{\max }}{\Delta \tau} & =\frac{\tau_{\text {turn }}}{\frac{4 k L}{(m+1) \Gamma(1 /(2 k))} \sqrt[2 k]{\log (m+1)}}+\frac{1}{2} \\
& =\tau_{\text {turn }} \frac{m+1}{4 k L} \frac{\Gamma(1 /(2 k))}{\sqrt[2 k]{\log (m+1)}}+\frac{1}{2} .
\end{aligned}
$$

Rewriting this condition in terms of the horizontal asymptote $L$,

$$
L<\frac{\tau_{\text {turn }}(m+1)}{2 k(2 n-1)} \frac{\Gamma(1 /(2 k))}{\sqrt[2 k]{\log (m+1)}} .
$$

The payoff extrema difference: We now calculate the payoff extrema difference (PED), $\Delta \Psi$, that is, the difference between a mutant's local minimum and maximum fitnesses when residents contribute the infinite-population ESS.

$$
\begin{aligned}
\Delta \Psi & =\left[B\left(\tau_{\max }\right)-\frac{\tau_{\max }}{n}\right]-\left[B\left(\tau_{\min }\right)-\left(\tau_{\min }-\frac{n-1}{n} \tau_{\max }\right)\right] \\
& =B\left(\tau_{\max }\right)-B\left(\tau_{\min }\right)-\left(\tau_{\max }-\tau_{\min }\right) \\
& =B\left(\tau_{\max }\right)-B\left(\tau_{\min }\right)-\Delta \tau,
\end{aligned}
$$

so using equations (60) and (63), we have

$$
\Delta \Psi=2 L \operatorname{erf}_{2 k}(\sqrt[2 k]{\log (m+1)})-\frac{4 k L}{(m+1) \Gamma(1 /(2 k))} \sqrt[2 k]{\log (m+1)}
$$

The mean fitness slope: To choose parameter values that generate a fitness difference with a distinct peak at the ESS (when residents play the ESS), we would like to find the mean fitness slope between the extrema, i.e., the ratio of the $\mathrm{PED}, \Delta \Psi$, and the distance between the fitness extrema as a function of our parameters. To that end, using equation (70), the distance between the fitness extrema is

$$
\frac{\tau_{\max }}{n}-\left(\tau_{\min }-\frac{n-1}{n} \tau_{\max }\right)=\tau_{\max }-\tau_{\min }=\Delta \tau .
$$

Equations (60) and (74) then yield

$$
\begin{aligned}
\frac{\Delta \Psi}{\Delta \tau} & =\frac{2 L \operatorname{erf}_{2 k}(\sqrt[2 k]{\log (m+1)})}{(m+1) \Gamma(1 /(2 k))} \sqrt[2 k]{\log (m+1)}-1 \\
& =(m+1) \frac{\Gamma(1 /(2 k))}{2 k} \frac{\operatorname{erf}_{2 k}(\sqrt[2 k]{\log (m+1)})}{\sqrt[2 k]{\log (m+1)}}-1,
\end{aligned}
$$


which only depends on the maximal marginal fitness, $m$ (and the order of the generalized error function, $2 k$ ). Note also that using equation (48) and L'Hôpital's rule (49),

$$
\lim _{x \rightarrow 0} \frac{\operatorname{erf}_{\ell}(x)}{x}=\lim _{x \rightarrow 0} \frac{\ell}{\Gamma(1 / \ell)} e^{-x^{\ell}}=\frac{\ell}{\Gamma(1 / \ell)},
$$

SO

$$
\lim _{m \rightarrow 0} \frac{\Delta \Psi}{\Delta \tau}=0 .
$$

In addition, equation (49) implies that for any $x>0$,

$$
\lim _{k \rightarrow \infty} \operatorname{erf}_{\ell}(x)=1
$$

(because $\Gamma(x) \rightarrow \infty$ as $x \rightarrow 0$, and $\Gamma\left(\frac{1}{\ell}, x^{\ell}\right)$ is bounded), and

$$
\lim _{x \rightarrow 0} x \Gamma(x)=\lim _{x \rightarrow 0} \Gamma(x+1)=1
$$

so we have

$$
\lim _{k \rightarrow \infty} \frac{\Delta \Psi}{\Delta \tau}=m
$$

The ratio of ESSs in infinite and finite populations: Using equations (60), (61) and $(67)$,

Rewriting the population size as $N=n G$,

$$
\begin{aligned}
\frac{X_{\infty}^{*}}{X_{N}^{*}} & =\frac{\tau_{\text {turn }}+\frac{2 k L}{(m+1) \Gamma(1 /(2 k))} \sqrt[2 k]{\log (m+1)}}{\tau_{\text {turn }}+\frac{2 k L}{(m+1) \Gamma(1 /(2 k))} \sqrt[2 k]{\log \left((m+1) \frac{N-n}{N-1}\right)}} \\
& =\frac{2 \tau_{\text {turn }}+\Delta \tau}{2 \tau_{\text {turn }}+\Delta \tau \sqrt[2 k]{\frac{\log \left((m+1) \frac{N-n}{N-1}\right)}{\log (m+1)}}} \\
& =\frac{2 \tau_{\text {turn }}+\Delta \tau}{2 \tau_{\text {turn }}+\Delta \tau \sqrt[2 k]{1+\frac{\log \left(\frac{N-n}{N-1}\right)}{\log (m+1)}}}
\end{aligned}
$$

We see that the ratio $X_{\infty}^{*} / X_{N}^{*} \rightarrow 1$ as $G \rightarrow \infty$ with $n$ fixed. However, $X_{\infty}^{*} / X_{N}^{*}$ approaches a (finite) value greater than 1 as $n \rightarrow \infty$ with $G$ fixed (assuming $X_{N}^{*}$ exist for all $N$; see inequality (15)). 


\section{B.2 Sigmoid using standard error-function}

In the special case $k=1$ (i.e., $\ell=2$ ), since $\Gamma(1 / 2)=\sqrt{\pi}$, equation (47) reduces to

$$
B(\tau)=L \operatorname{erf}\left((m+1) \frac{\sqrt{\pi}}{2 L}\left(\tau-\tau_{\text {turn }}\right)\right), \quad \tau \geq 0
$$

Then, setting $k=1$ in equation (85) gives the maximizing and minimizing total goods,

$$
\begin{aligned}
& \tau_{\text {min }}=\tau_{\text {turn }}-\frac{2 L}{m+1} \sqrt{\frac{\log (m+1)}{\pi}}, \\
& \tau_{\max }=\tau_{\text {turn }}+\frac{2 L}{m+1} \sqrt{\frac{\log (m+1)}{\pi}},
\end{aligned}
$$

and the distance between the location of the fitness minimum and maximum is

$$
\Delta \tau=\tau_{\max }-\tau_{\min }=4 \frac{L}{m+1} \sqrt{\frac{\log (m+1)}{\pi}} .
$$

Equation (61) then gives

$$
X_{\infty}^{*}=\frac{1}{n}\left(\tau_{\text {turn }}+\frac{2 L}{m+1} \sqrt{\frac{\log (m+1)}{\pi}}\right),
$$

and equation (62) becomes

$$
b_{\text {curve }}=B^{\prime \prime}\left(\tau_{\max }\right)=-\frac{m+1}{L} \sqrt{\pi \log (m+1)} .
$$

From equation (63),

$$
B\left(\tau_{\max }\right)-B\left(\tau_{\min }\right)=2 L \operatorname{erf}(\sqrt{\log (m+1)})
$$

Equation (67) gives the unique $\mathrm{ESS}_{\mathrm{N}}$ :

$$
X_{N}^{*}=\frac{1}{n}\left(\tau_{\text {turn }}+\frac{2 L}{m+1} \sqrt{\frac{\log \left((m+1) \frac{N-n}{N-1}\right)}{\pi}}\right),
$$

and equation (68) becomes

$$
B^{\prime \prime}\left(n X_{N}^{*}\right)=-\frac{m+1}{L} \sqrt{\pi \log \left((m+1) \frac{N-n}{N-1}\right)} .
$$

Condition (71), which guarantees that when a single mutant invade a population playing the ESS, the fitness difference has both a minimum and a maximum (as a function of the mutant strategy), reduces to

$$
n<\tau_{\max } / \Delta \tau=\frac{\tau_{\text {turn }}}{4} \frac{m+1}{L} \sqrt{\frac{\pi}{\log (m+1)}}+\frac{1}{2},
$$


861

the PED, $\Delta \Psi$ (equation (74)) becomes

$$
\Delta \Psi=2 L \operatorname{erf}(\sqrt{\log (m+1)})-4 \frac{L}{m+1} \sqrt{\frac{\log (m+1)}{\pi}},
$$

and the mean fitness slope (equation (76)) between the extrema reduces to

$$
\frac{\Delta \Psi}{\Delta \tau}=\frac{m+1}{2} \sqrt{\frac{\pi}{\log (m+1)}} \operatorname{erf}(\sqrt{\log (m+1)})-1 .
$$

